\title{
Diversity of Phylogenetic Information According to the Locus and the Taxonomic Level: An Example from a Parasitic Mesostigmatid Mite Genus
}

\author{
Lise Roy $^{1, *}$, Ashley P. G. Dowling ${ }^{2}$, Claude Marie Chauve ${ }^{1}$ and Thierry Buronfosse ${ }^{1}$ \\ 1 Laboratoire de Parasitologie, Université de Lyon, Ecole Nationale Vétérinaire de Lyon, \\ Marcy-L'Etoile, France; E-Mails: c.chauve@vet-lyon.fr (C.M.C.); \\ thierry.buronfosse@inserm.fr (T.B.) \\ 2 Department of Entomology, University of Arkansas, Fayetteville, USA; \\ E-Mail: adowling@uark.edu \\ * Author to whom correspondence should be addressed; E-Mail: 1.roy@vet-lyon.fr; \\ Tel.: + 6-69-78-76-87; Fax: + 4-78-87-25-77.
}

Received: 5 March 2010; in revised form: 8 April 2010 / Accepted: 8 April 2010 /

Published: 13 April 2010

\begin{abstract}
Molecular markers for cladistic analyses may perform differently according to the taxonomic group considered and the historical level under investigation. Here we evaluate the phylogenetic potential of five different markers for resolving evolutionary relationships within the ectoparasitic genus Dermanyssus at the species level, and their ability to address questions about the evolution of specialization. COI provided $9-18 \%$ divergence between species (up to $9 \%$ within species), $16 \mathrm{~S}$ rRNA $10-16 \%$ (up to $4 \%$ within species), ITS1 and $22-9 \%$ (up to $1 \%$ within species) and Tropomyosin intron $n$ $8-20 \%$ (up to $6 \%$ within species). EF-1 $\alpha$ revealed different non-orthologous copies within individuals of Dermanyssus and Ornithonyssus. Tropomyosin intron $\mathrm{n}$ was shown containing consistent phylogenetic signal at the specific level within Dermanyssus and represents a promising marker for future prospects in phylogenetics of Acari. Phylogenetic analyses revealed that the generalist condition is apomorphic and D. gallinae might represent a complex of hybridized lineages. The split into hirsutus-group and gallinaegroup in Dermanyssus does not seem to be appropriate based upon these results and $D$. longipes appears to be composed of two different entities.
\end{abstract}


Keywords: phylogenetic signal; evolution of specialization; Dermanyssus; Acari; Mesostigmata

\section{Introduction}

The question of the evolution of ecological specialization is a fundamental one that may be partly addressed through phylogenetic analyses. Habitat or resource range may be dictated partly by extrinsic ecological parameters (biotic and/or abiotic environmental factors) and partly by intrinsic characteristics. The specialist/generalist condition may be imposed by ecological factors such as competition and mating success, or simply may be historically acquired. As a result, phylogenetic reconstructions are expected to help assess the evolution of specialization in a given group.

It has been often presumed that specialists evolve from generalists [1], but several recent studies involving host specificity in host-parasite systems [2], food resources in bees [3], or habitat in springtails [4] show generalists in distal positions. In these examples, specialization appears to be plesiomorphic and does not appear to be a "dead end". Futuyma and Moreno [1] pointed out that the irreversibility of specialization is more likely to occur when phenotypes have been highly modified (reduced, lost characters). In such cases, specialization is likely to strongly restrict evolutionary flexibility. On the other hand, generalization may have a cost [5] also restricting evolutionary plasticity.

It has been commonly assumed that specialized species, because of their narrow tolerance and consequently fragmented distribution, are more prone to allopatric speciation than are generalists [1]. As a result, reduced gene flow between specialized lineages might contribute to fixation of characters which otherwise would have been lost in the framework of extensive outbreeding (which generates an increased effective population size). But Kaci-Chaouch et al. [6] showed that generalist species within the genus Lamellodiscus, which are more derived diplectanid monogeneans [2], have more genetic and morphological diversity than specialists. This contradicts the hypothesis of a positive relationship between specialization and rate of diversification.

The mite genus Dermanyssus is involved in a rather loose host-parasite system and therefore represents an interesting model for testing the trends in the evolution of specialization. Following Kuris and Lafferty [7], Dermanyssus species in the gallinae-group sensu Moss [8] are considered micropredators rather than typical parasites because adult females feed successively on different host individuals like mosquitoes or bed bugs. Blood meals are taken rapidly resulting in engorgement [9] and are limited in number (one per nymphal stage and one before each oviposition in adult females). Members of the gallinae-group are nidicolous in nature, laying eggs and spending much time off the host. In contrast, species in the hirsutus-group are more typical ectoparasites, living and developing on the host [8].

Despite the loose host relationship in the gallinae-group, they exhibit various levels of host specificity. Some species that had previously been considered to have a very broad host range turned out to be more specific than thought, likely due to previous misidentifications [10]. Roy et al. [10,11] highlighted an opposition between two main clades, which seemed to be correlated with the level of 
host specificity. On one hand, there was the D. gallinae clade with generalist species (nine different bird orders), including diverse lineages, of which some may by potentially cryptic species. On the second hand there was a clade of four specialist species $(D$. hirundinis, Passeriform hosts, a single bird family in France, D. longipes, Passeriform hosts, 2 bird families, D. carpathicus, id., and D. apodis, Apodiform host, a single bird genus) [10]. Moreover, D. gallinae is the only synanthropic species (encountered in bird farms). Although the studies of Roy et al. [10,11] reached a solid delineation between species, they did not succeed in resolving relationships between specific entities. This was due to the use of mt DNA data only [10] or to the weakly informative variations in the selected nDNA fragment (ITS1-5.8S-ITS2) [11].

The present study has the following two objectives:

(1) Assess the utility of two additional nuclear markers - elongation factor 1- $\alpha$ (EF-1 $\alpha)$, Tropomyosin exon $n$, intron $n$ and exon $n+1(T p m)$ - for the exploration of relationships between and within species belonging to Dermanyssus and comparing these results to previous studies $[10,11]$

(2) Address the following questions about the evolution of host specificity in Dermanyssus:

a Are the lineages of the generalist $D$. gallinae's lineages effectively composed of cryptic species (potentially making them as specialized as any of the four specialist species)?

b Is the generalist condition derived or ancestral?

For such purposes, we performed phylogenetic analyses based on a combination of different gene fragments. Analyses aim to not only disentangle relationships between species but also to further explore species delineations (i.e., check the reproductive status of some mitochondrial lineages previously noticed within delineated entities). Therefore, the degree of coalescence of some intraspecific clades was compared between mt and nDNA topologies and a comparison of observed intraspecific variability was carried out.

\section{Material and Methods}

Usually, in order to improve phylogenetic analyses, increasing the number of characters or the number of taxa may be helpful. In the present study, we adopt a two-step strategy: first, a multi-gene analysis was performed on a reduced set of isolates, based on five different genes representing six different datasets (Step 1). Then a multi-isolate analysis was performed using one mitochondrial and one nuclear marker developed in the first stage (Step 2), since less isolated lineages were detected in previous DNA studies [10,11]. This second approach focused on intraspecific and intra-isolate variability, by using sequences of two independent and highly variable loci. Although not expected to fill in taxonomic gaps, this was expected to provide finer resolution of relationships within previously delimited specific entities in order to enable detection of cryptic isolated lineages [11]. The focus is on the five following species: D. gallinae, D. apodis, D. carpathicus, D. hirundinis, D. longipes.

\subsection{Biological Material}

The location, host species, mite species, accession numbers of mites under test are listed in Appendix 1. Mite isolates have been sampled from wild bird nests or from farms as described in Roy et al. [10]. The distribution of samples is rather large and diverse within France, and includes a few 
samples from other countries (ex. The Netherlands, Poland, USA)(Appendix 1). Nests were analyzed using a method described by De Lillo [12] involving immersion of the nest followed by filtering, except that no sodium hypochlorite was added to the water solution to wash the stack of sieves and that the sieves had a somewhat different mesh width (top to bottom: $2,500 \mu \mathrm{m}, 1,400 \mu \mathrm{m}, 180 \mu \mathrm{m}$, $100 \mu \mathrm{m})$.

One isolate corresponds to mites of a single Dermanyssus species, isolated from an individual nest or from a group of nests closely located to each other in a bird colony (wild avifauna) or from a single building (farms). From each isolate, 1-5 individuals have been separately sequenced. Additionally, for five of these isolates $(1 \mathrm{D}$. apodis: GO, 1 D. carpathicus: BER7, 2 D. gallinae s. str.: SK, IL, 1 D. gallinae special lineage L1: 9001; see Roy et al. 2009a), 18-24 individuals have been separately sequenced, in order to get an overview of the intrapopulation variation. Moreover, 21 individuals belonging to D. hirundinis collected from barn swallows distributed around France were included in the analyses and are referred to as DhirF. These six groups are used specifically for statistical analyses.

\subsection{DNA Data}

Data are composed of DNA sequences obtained following the method described in Roy et al. $[10,11]$ and newly obtained DNA sequences following the procedures below. The previously tested DNA regions include partial 18S-28S rRNA, including complete ITS1, ITS2 and 5.8 S (ITS), partial 16S rRNA and partial coding gene for cytochrome oxidase I (COI). The newly developed markers are one intron flanked by two portions of the coding gene for Tropomyosin and a portion of the coding gene for elongation factor 1-alpha $(\mathrm{EF}-1 \alpha)$.

Sequences of Tropomyosin and EF-1 $\alpha$ were obtained as in Roy et al. [11], with PCR involving annealing temperatures of $56{ }^{\circ} \mathrm{C}$ and $57{ }^{\circ} \mathrm{C}$, respectively. Primer sequences and pairs are provided in Appendix z2.

D. gallinae has been shown to be haplodiploid with diploid females developing from fertilized eggs $[13,14]$. As these authors also observed similar haplodiploidy in a closely related family (Macronyssidae), we assume here that other Dermanyssus species reproduce the same way. As a result, only adult females were integrated in the study for standardization, notably to regularly detect allelic variation and to avoid potentially confounding effects of the male haploid genome.

In case of heterozygosity in the Tropomyosin targeted fragment, the two alleles were separated from each other using internal primers (cf. Appendix z2), or in some cases by cloning. As for EF-1 $\alpha$ some sequences were cloned to separate double copies found after PCR. Vector cloning was necessary for separation of multi-copies sequences in some cases. PCR products were gel purified (Qiagen, Les Ulis France) and corresponding fragments were cloned into TOPO ${ }^{\circledR}$ TA cloning vector according to manufacturers' protocols. Transformant clones were checked by restriction enzyme profile and five positive clones were submitted to sequencing.

\subsection{Datasets}

DNA and amino acid alignments were performed using MUSCLE 3.7. Without refinement, MUSCLE has been shown to achieve accuracy statistically indistinguishable from T-Coffee and MAFFT, but overall is the fastest of the tested methods for large numbers of sequences [15]. Seaview 
4.0 [16] was used for DNA and amino-acid alignment handling. Six different matrices, composed of DNA sequence alignments, were generated during Step 1 and three different matrices, two of which are DNA sequence alignments and one is a digital matrix, during Step 2.

\section{Step 1: multi-gene analyses}

Six matrices are composed of assumed independent data as defined by Li and Lecointre [17]. The matrix combining the six datasets as labeled in Table 1 is available in Treebase (ID number 10389).

Table 1. Elementary datasets.

\begin{tabular}{lll}
\hline \multicolumn{1}{c}{ mtDNA } & \multicolumn{1}{c}{ nDNA } \\
\hline rRNA & 1 comb2 $(16 \mathrm{~S})$ & 1 comb3 (5.8S) \\
Internal Transcribed Spacers & & 1 comb4 (ITS1 \& ITS2) \\
Protein coding genes & 1 comb1 (COI) & 1 comb6 (Tropomyosin exon $\mathrm{n} \& \mathrm{n}+1, \mathrm{EF}-1 \alpha)$ \\
Intron & & 1 comb5 (Tropomyosin intron $\mathrm{n})$ \\
\hline
\end{tabular}

\section{Step 2: multi-isolate analyses}

Haplotype alignments for Tropomyosin and COI include data from 257 individuals, representative of 40 isolates of $D$. gallinae (146 individuals), 6 isolates of $D$. carpathicus (40 individuals), 2 isolates of $D$. apodis (25 individuals), 10 isolates of $D$. hirundinis (22 individuals), 3 isolates of $D$. longipes (8 individuals), plus 1 individual of $D$. hirsutus, 1 of $D$. quintus and 14 of outgroup species (see Appendix 1 and Treebase).

Additionally, in order to test the phylogenetic utility of indels in Tropomyosin intron $\mathrm{n}$, a matrix of indels in the Tropomyosin intron $\mathrm{n}$ has been elaborated by encoding as discrete characters the presence/absence and, when present, polymorphism of inserts at points where gaps are noted in the alignment of the whole Tropomyosin dataset. These indels have been encoded as if they were morphological or biochemical characters (character states and matrix are available in Appendix z3).

The haplotype alignments of COI and Tpm are available in Treebase (ID number 10389).

\subsection{Phylogenetics}

\subsubsection{Phylogenetic Analyses}

Phylogenetic analyses with Maximum parsimony (MP) were run using PAUP* 4.0b10 under the same parameters as in Roy et al. [11]. In order to explore phylogenetic information from indels, MP analyses were performed using either the encoded indels matrix or sequence alignments with gaps as missing data or as a fifth state.

In addition to parsimony, Bayesian analyses were run using MrBayes incorporating the most appropriate model of evolution for each dataset as determined in MrModeltest using Akaike information criterion as in Roy et al. [11]. Since MrBayes allows each partition to be simultaneously analyzed under a separate model of evolution, each dataset was analyzed with its most appropriate model (COI: GTR + G + I; 16S: GTR + G; 5.8S, ITS, Tropo_int, Tropo_ext: HKY + I). In cases of single gene analyses, only the most appropriate model of evolution for each dataset was used in the 
respective analysis. Parameters within the model were not specified (or fixed) and MrBayes was left to estimate these independently from the data during analysis. Analyses in MrBayes included two independent runs, each consisting of four chains and 10,000,000 generations for the total combined dataset and 2,000,000 generations for each of the independent datasets. Appropriate burnins were determined based on stationarity being reached through the use of Tracer v1.4 [18].

\subsubsection{Outgroups}

Trees were rooted using the outgroup method. Outgroup mites sequenced include Ornithonyssus sylviarum and $O$. bacoti (Parasitiformes: Mesostigmata: Dermanyssoidea Macronyssidae), Androlaelaps casalis (Parasitiformes: Mesostigmata: Dermanyssoidea: Laelapidae), and Typhlodromus pyri (Parasitiformes: Mesostigmata: Ascoidea: Phytoseiidae). Additionally, some sequences have been sampled from EMBL database for two other mesostigmatid mites, Tropilaelaps (accession numbers: ITS EF02474, COI EF025423) and Varroa (accession numbers: ITS EF025470, COI and 16S NC_004454).

Concerning EF-1 $\alpha$, due to apparent paralogy and in order to roughly estimate the divergence point between some of the obtained paralogous copies, more distant outgroups have been included (additional sequences drawn from EMBL bank, other Parasitiformes plus some Acariformes; accession numbers: EU152805, EU152810, EU152811, EU152815, EU152816, EU152823, EU152832, EU152837, EU152840, EU152844, EU152853, U90048, AY624011, AF240836, AF240849, AF240856, AF240851, AF240860, AY624008, AY624009, AAT58070). Note that outgroup method used here for tree rooting is not an ancestry method. The aim of outgroup inclusion is simply to check the target group's monophyly and explore its internal evolutionary history.

Lastly, although variously distant outgroups have been included into analyses, no very close outgroup have been sampled. Dermanyssidae currently include two genera, Dermanyssus and Liponyssoides. Despite repeated attempts to collect species of Liponyssoides in its typical host mammals, we did not manage to find any individuals of this genus. As a result, in the present study, monophyly of Dermanyssidae is tested, but the monophyly of Dermanyssus is not.

\subsubsection{Clade Robustness Support Values}

Two different methods for estimating clade support have been used here: classical node support values (bootstrap, BPP) in Steps 1 and 2, as well as a newly developed index [17] in Step 1. Indeed, in order to avoid the problem of stochastic effects of homoplasy in single gene analyses, the method proposed by Li and Lecointre [17] offers the opportunity to simultaneously estimate robustness of both single gene and combined analyses. This consists of observing clades obtained using all partial or total combination possibilities in partitioning schemes which each includes all the elementary datasets and noting their occurrence. A repetition index is drawn from it.

For such a purpose, phylogenetic reconstructions of elementary datasets and of all possible combinations (two to six elementary datasets) were computed using PAUP 4.0 (MP) (with 1,000 random additions instead of 10,000). Notation of clade occurrence based on each $50 \%$ majority rule consensus tree, calculation of all possible partitioning schemes and automation of the consecutive 
estimation of repetition indices following $\mathrm{Li}$ and Lecointre [17] were performed using macro functions (VBA) in Excel.

\subsubsection{Comparison of Mitochondrial versus Nuclear Monophylies}

Due to some differences in the effective population size between mitochondrial and nuclear DNA evolution, the monophyly of alleles is expected to appear more quickly in mt-DNA than in nuclear DNA. In most sexually reproducing animals (diplodiploids), cytoplasmic DNA is effectively haploid and maternally inherited, as opposed to nuclear DNA, which is diploid and biparentally transmitted. Consequently, they have a genetically effective population size approximately four times smaller than that of nuclear loci [19]. In the present case, the ratio is reduced to three times due to the haplodiploidy of D. gallinae (see above). In order to estimate the degree of structure within the newly developed nuclear marker Tropomyosin, the ratio external/internal branch length in the Bayesian mitochondrial toplogy (Step 2) has been observed in delimited specific entities following Roy et al. [11] and in some additional intraspecific clades, and correlated with monophylies in the Tropomyosin topologies.

Additionally, in order to detect isolated lineages within species boundaries, the most supported lineages in Step 2 (both $\mathrm{mt}$ and nDNA) have been examined by mapping isolates on the haplotypic topologies and by comparing the amount of shared isolates.

\subsection{Statistical Analysis of Haplotype Frequencies and Diversity}

Some statistical analyses were performed between and within the six focused isolates, representing three species. They were performed using the isolate DNA alignments of COI haplotype sequences on one hand, and individual Tropomyosin alleles (phased alleles in heterozygous individuals and duplicated homozygous sequences, in such a way that sequences represent the diploid state of chromosomes) on the other hand.

Polymorphism in haplotype sequences (COI haplotypes and separated Tropomyosin alleles) within the eight "focused isolates" was examined (gaps excluded and as the fifth state in Tropomyosin) using DnaSP v5 [20]. We estimated the number of segregating sites (S), average number of nucleotide differences $(\mathrm{k})$, and haplotype diversity $(\mathrm{Hd})$. Pairwise genetic distances were computed using Fst (Hudson et al. 1992) and statistical significance assessed after 1000 permutations in all cases using Arlequin 3.1 [21] (haplotypic dataset for COI and genotypic dataset for Tropomyosin in order to avoid potential bias due to departure to the Hardy-Weinberg equilibrium).

\section{Results}

\subsection{DNA Sequences}

\subsubsection{Alignments of Obtained Gene Fragments}

Three DNA alignments are available in Treebase (accession number: 10389): the matrix 6comb combining the six elementary datasets and corresponding to the partitioning scheme psc 203 (Appendix 4) of Step 1 and the COI and the Tpm haplotypic alignments of Step 2. 
Obtained sequences of rRNA, internal transcribed spacers and intron show variable lengths: $16 \mathrm{~S}$ rRNA and 5.8S rRNA, ITS1 and ITS2, Tropomyosin intron n. Other sequences are strictly composed of gene portions coding for proteins and did not include stop codons nor indels: COI, EF-1 $\alpha$, Tropomyosin exons $\mathrm{n}$ and $\mathrm{n}+1$.

In $16 \mathrm{~S}$ rRNA, a portion corresponding to a stem-loop structure known to be highly variable in some ticks if compared to Drosophila yakuba (between positions 200 and 255 in Black and Piesman [22]) shows variability between specific entities including numerous indels. Indels being correlated to the secondary structure in this region, the unambiguity of alignment in this portion is not established. However, excision or not of this portion did not change present results.

ITS1 and 2 are amazingly stable within and between species within genera compared to some other mites (cf. Below, Section 3.2.2). As a result, they are unambiguously aligned within genera.

In Tropomyosin intron $\mathrm{n}$, indels are numerous, but unambiguously aligned as already noted in two EF-1 $\alpha$ introns by Sanchis et al. [23] and by Kawakita [24].

No ambiguity has been detected in protein coding nucleic sequences.

\subsubsection{Molecular Characteristics of Obtained Gene Fragments}

Fragments of 16S rRNA, COI and ITS obtained in the present study correspond to those used by Roy et al. [10,11].

The nuclear EF-1 $\alpha$ gene fragment corresponds to positions 303-891 of the Heliconius melpomene (Insecta:Lepidoptera) EF-1 $\alpha$ gene and 102-298 of the H. melpomene protein (complete CDS, accession number: GQ452009). All sequences were free of stop codons. Moreover, no intron has been isolated in any mesostigmatid individual under test in the present study.

Note: The homology of obtained EF-1 $\alpha$ sequences is far from certain. EF-1 $\alpha$ copies obtained from Ornithonyssus and Dermanyssus group into two different clades containing both genera, not only when analysis is processed on nucleic sequences (clades EF-A and EF-B in Figure 1), but also on translated amino acid sequences (results not shown). Multiple copies of EF-1 $\alpha$ belonging to the two clades EF_A and EF_B have been detected in single individuals (O. sylviarum FS5, FS6, D. gallinae ROL09) It is likely that most individuals contain similar multiple copies, although only a few of them amplified multiple copies in a single PCR run, due to primer mismatch or competition as already noted in some spiders by Hedin and Maddison [25]. Moreover, there are sequences shared between some ingroups and outgroups (O. sylviarum PM and JBO10, D. gallinae - all isolates -, D. longipes PAS) in clade EF_B. Consequently, this locus has been discarded from elementary datasets and the matrix 1 comb6 has been reduced to Tropomyosin exon $n$ at $n+1$ alone (Table 1 ).

The nuclear Tropomyosin gene fragment involved in the whole analysis corresponds to $10 \mathrm{bp}$ of exon $\mathrm{n}$, a 585-664 bp intron $\mathrm{n}$ and 15 bp of exon $\mathrm{n}+1$. Intron $\mathrm{n}$ is located between positions 551 and 552 of the coding gene in Boophilus microplus, based on the complete CDS published in GenBank (AF124514) and between positions 490 and 491 of the CDS sequence of $D$. gallinae published by Nisbet et al. [26] (AM167555). In order to check the homology of aligned introns, larger Tropomyosin fragments from 1-2 individuals of four Dermanyssus species were first sequenced (individuals of D. apodis GO593 and MAR1, D. gallinae 8004b, D. carpathicus RQ18, D. longipes JBO49DL2; see 
species and EMBL accession number in Appendix 1). This way, five sequences, including a 62-115 bp portion of exon $n$, the focused intron and a 53-80 bp portion of exon $n+1$ were aligned.

Figure 1. Strict consensus of 95 equiparsimonious trees inferred from nucleotide EF-1 $\alpha$ sequences. Tree length $=1714, \mathrm{CI}=0.3215, \mathrm{RI}=0.6909$. Numbers at nodes correspond to bootstrap values (only values $>50 \%$ are indicated). The three following groups of sequences have been isolated in a single individual: O_sylviarum_FS6a and b, D_gallinae_ROL0951, 2, 3 and 4,D_gallinae_ROL0961, 2, 3 and 4. Trees are rooted using Hemialges as an outgroup. EF_A and EF_B represent a bipartition apparently generated by gene duplication.

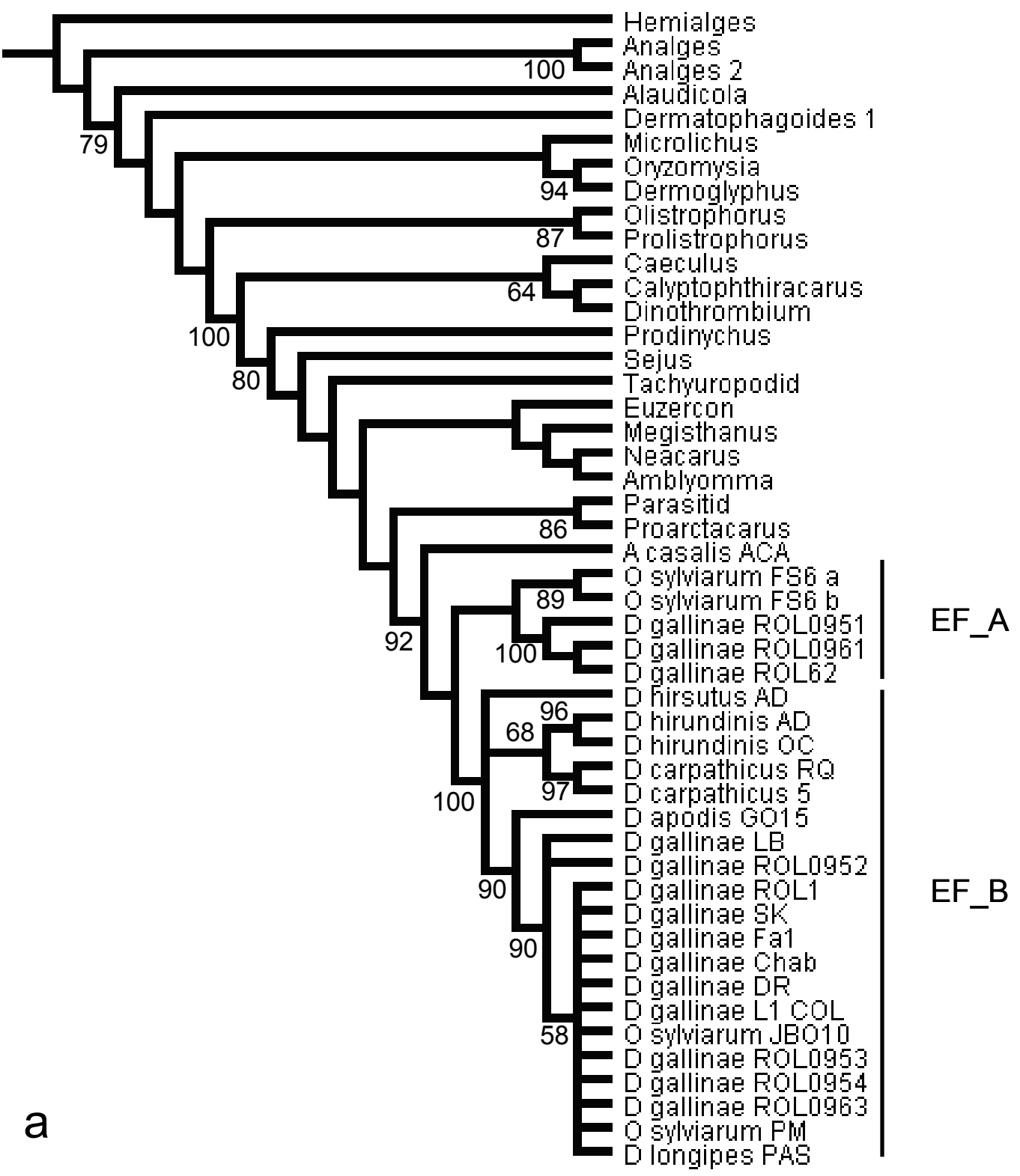

This allowed confirmation of homology. Additionally, an alignment was performed with the above sequences after the intron was removed. The portion of coding regions provided in the present study was exactly the same as the corresponding part in the D. gallinae CDS sequence in extended sequences of individuals of $D$. gallinae, D. carpathicus and D. longipes. In the sequences of the two 
individuals of $D$. apodis, a single nucleotide polymorphim in exon $\mathrm{n}$ and 1 in exon $\mathrm{n}+1$ was noted ("C" instead of "T" at position 489 (exon n) and at position 498 (exon $n+1$ ) of Nisbet et al's CDS). As for the translated amino acids sequences, they were free of stop codons, identical in all six Dermanyssus sequences and very close to B. microplus (differing by only three amino acids).

Within the intron, more than 50 sites involve indels, but in many cases a fixed series of 3-5 bp (and even up to $15 \mathrm{bp}$ ) is inserted/deleted, resulting in inserted/deleted 35 bp-portions in the whole dataset of gallinae-group individuals (see Appendix z3). One region involves some microsatellite motifs, whose number is strongly varying between species, between isolates and within isolates. Sites with indels have been recorded based on alignment ISOL_TRO1 (available online as ESI) and their distribution all along the region under test is located only on intron $\mathrm{n}$ and is rather regular when the five focused species are included (Figure 2). The first hundred and the last hundred base pairs are free of indels. Several indels were also detected within D. gallinae, which allowed easy separation of alleles in cases of heterozygosity. Important regions with indels have also been noted within D. longipes (ID3, ID4, C1, C2, cf. Appendix z3).

Note that indels are found mainly in addition in D. gallinae and D. apodis (and in subtraction in D. longipes, D. hirundinis, D. carpathicus). As a result, sequences of D. apodis and D. gallinae populations are longer than in D. longipes, D. hirundinis, D. carpathicus (670-695 bp vs. $615-652$ bp).

Figure 2. Overview of the distribution of variable sites along the studied Tropomyosin sequence (mutation points and indel regions) on the basis of alignment ISOL_TRO1 (available online as electronic supplementary information (ESI)). Point mutations are figured by open triangles, indels detected in the whole Dermanyssus dataset by black dots and indels within $D$. gallinae only by grey dots. Uppercases refer to indel regions within D. gallinae as labeled in Appendix z3.

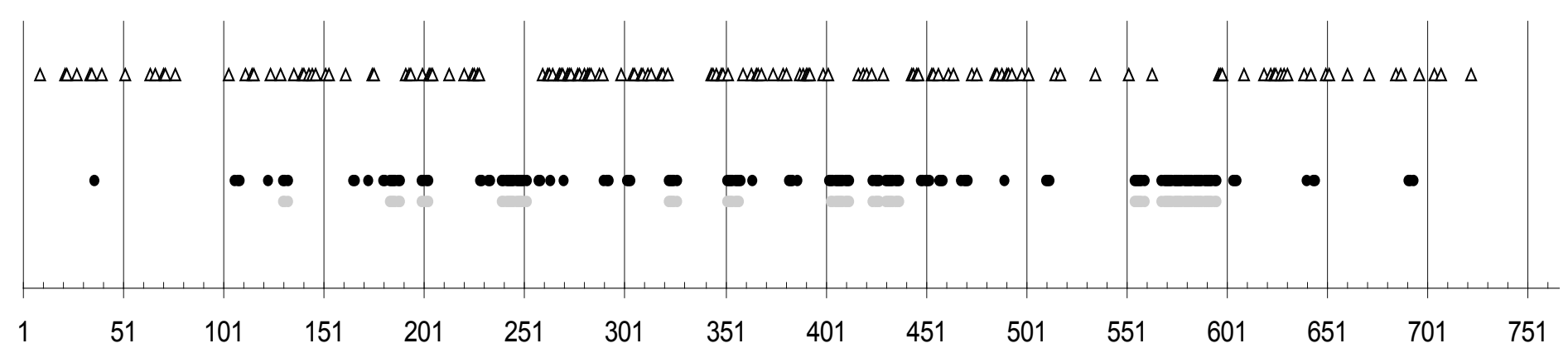

\subsection{Step 1: Multi-Gene Analyses}

The five gene portions have been obtained in six Dermanyssus species, two Ornithonyssus species and one Androlaelaps species. Within Dermanyssus, one isolate of D. hirsutus, two of $D$. apodis, two of $D$. longipes, three of $D$. hirundinis, three of $D$. carpathicus, nine of $D$. gallinae (of which three of D. gallinae special lineage L1, see Roy et al. [11]) have been integrated into the analyses. In some cases, two different profiles have been distinguished within some isolates of D. gallinae and 
D. carpathicus, due to some intra-isolate variations (mutations and/or indels) in COI and or Tropomyosin sequences. See Appendix 1 for detailed informations about individuals, isolate locations and accession numbers. All genes except Tropomyosin have been found for one additional dermanyssoid genus in EMBL database (Varroa) and all except Tropomyosin and 16S for another one (Tropilaelaps).

\subsubsection{Phylogenetic Interrelationships at the Specific Level}

In addition to the six elementary datasets, there were 57 different possible combinations of two to six elementary datasets resulting in 203 different partitioning schemes following Li and Lecointre [17] (see Appendix 4).

On the whole, 24 clades have been observed (see Table 2), and 38 different topologies, of which six exhibit mostly unresolved relationships (see Appendix 5). Bayesian analyses of elementary datasets as well as of the fully combined dataset (psch 203) resulted in similar topologies (results not shown).

The method of $\mathrm{Li}$ and Lecointre aims at reducing the stochastic effects of homoplasy, which increases as the dataset's size decreases, especially in single gene analyses. Nevertheless, the more a clade occurs within partitioning schemes, i.e., the less combination is needed to get it retained, the more it is considered reliable. Two clades were recovered in analysis of each individual dataset, one grouping together all isolates of Dermanyssus $(\pi)$ and the other grouping all isolates of Ornithonyssus $(\sigma)$. Three of the five specific entities with several isolates under test generate clades that occur up to five times per partitioning schemes (D. apodis, D. carpathicus, D. hirundinis), as well as the special lineage L1 of $D$. gallinae. Isolates of $D$. gallinae and of $D$. longipes group together up to 4 times per partitioning scheme.,No contradictory evidence has been noted for D. gallinae monophyly (only unresolved relationships lead to unrecovered clade in some cases), whereas contradictions have been noted for $D$. longipes monophyly (clades $\gamma$ and o in Table 2). Consequently, the analysis of specific level relationships takes separately into account D. longipes EN and D. longipes PAS, D. gallinae L1 and D. gallinae non L1. Analyses of elementary datasets 1 comb3 (5.8S) and 1 comb6 (Tropomyosin exon $n$ and $n+1$ ) did not recover any of the specific entities. The elementary dataset 1 comb1 (COI) recovered all species under test. The elementary dataset 1comb2 (16S) recovered all species except D. gallinae. The elementary dataset 1 comb4 (ITS1 and 2) recovered all species except D. gallinae and D. apodis. The elementary dataset 1 comb5 (Tropomyosin intron n) recovered all species except D. longipes.

Finally, relationships between specific entities within Dermanyssus are supported by clades with three to four occurrences per partitioning scheme.

By examining the percentage of occurrence of topologies retained based on $50 \%$ majority rule consensus trees among the 203 different partitioning schemes and the maximum number of their occurrences, and by considering the level of their resolution, it appears that among the most resolved topologies, Top2, Top17 and Top7 are recurrently encountered with both gaps as missing data and gaps as the fifth state, and they appear up to twice in a single partitioning scheme (see Appendix 4). Of them, the first two are very close to each other. Top2 is fully resolved, with D. gallinae L1 as a sister to other D. gallinae isolates, whereas Top17 has D. gallinae L1 branching from within other isolates of D. gallinae. Top7 is close to Top17, except that it presents D. longipes as paraphyletic. 
The greedy consensus with gaps as missing data is exactly Top2 (Figure 3). And the greedy consensus obtained with gaps treated as the fifth state is closest to Top 10, i.e., it resembles Top2, with the exception that $D$. hirsutus is transferred from the most basal position to the median position of sister to clade $\xi$ (D. gallinae + D. apodis). And yet, the total evidence (6comb, partitioning scheme $\mathrm{n}^{\circ}$ 203) Bayesian analysis results in Top12 (see Appendix 5), i.e., the strict consensus of both greedy consensuses above.

Figure 3. Trees summarizing the most repeated clades encountered in Step 1 (multi-gene analyses) at the specific level with the greedy summary method described in $\mathrm{Li}$ and Lecointre [17]. Maximum Parsimony criterion, PAUP 4.0. The maximum number of occurrence of the clades and the repetition indices (in bold) are above the branches. The bootstrap supports in the "total evidence" MP analysis are in bold below branches and the Bayesian Posterior Probabilities in the "total evidence" BA analysis are in italic below branches. (a) Analyses treating gaps as missing data. (b) Analyses treating gaps as the fifth state.
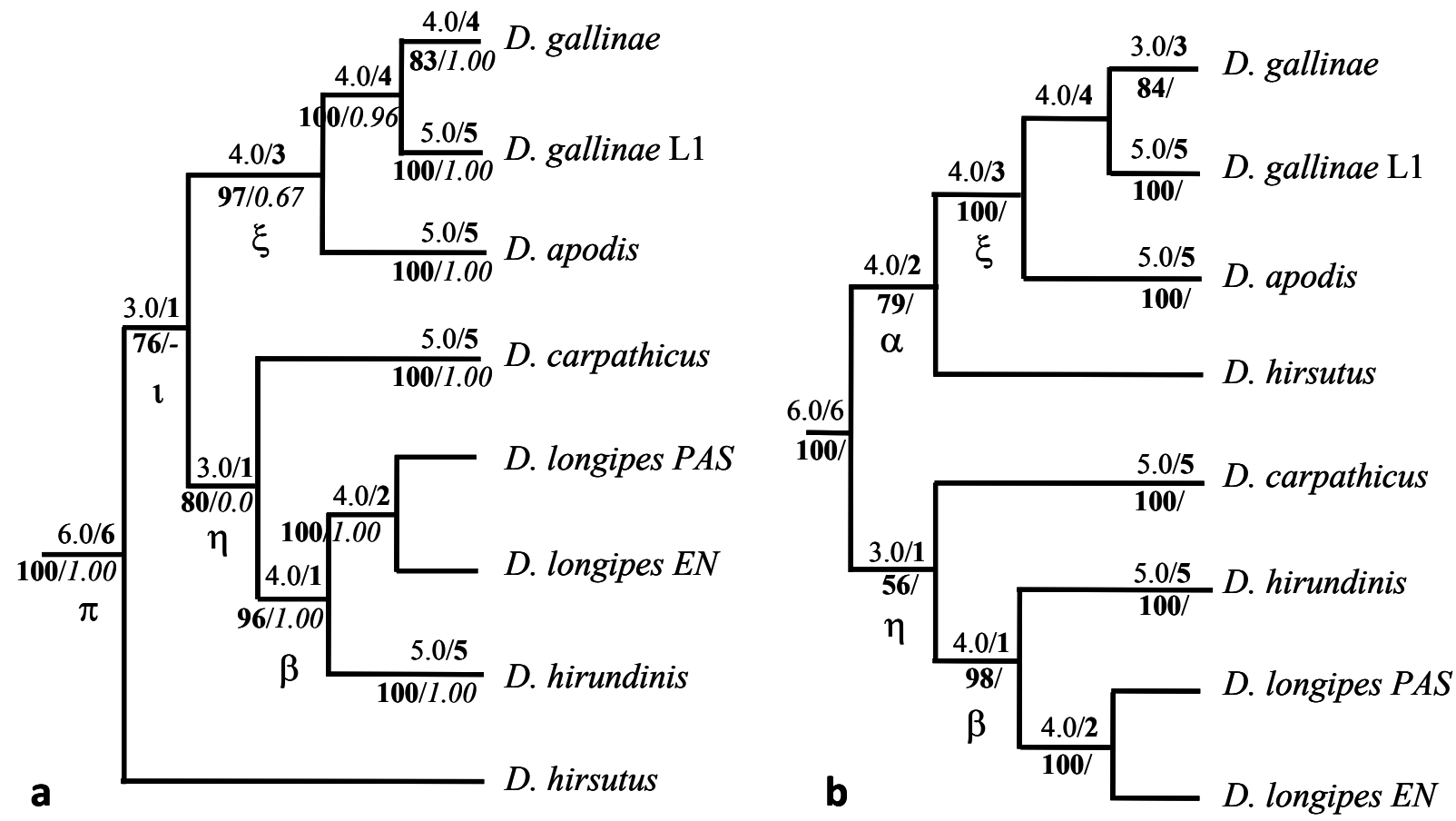
Table 2. List, composition and support values of clades recovered in any analyses in the Steps $\mathbf{1}$ and 2.

\begin{tabular}{|c|c|c|c|c|c|c|c|c|c|c|c|c|c|c|c|c|c|c|c|c|c|c|}
\hline & & & & & & Iso & te $n c$ & & & & & & multi- & gene & IP analy & & $\begin{array}{l}\text { multi-gene } \\
\text { MP COI }\end{array}$ & $\begin{array}{l}\text { multi-gene } \\
\text { BA COI }\end{array}$ & multi-gene $\mathrm{N}$ & MP Tropomyc & sin analysis & $\begin{array}{c}\text { multi-gene BA } \\
\text { Tropomyosin }\end{array}$ \\
\hline $\bar{U}$ & & & $\stackrel{0}{\simeq}$ & & & & & & & & & & $\begin{array}{r}\text { Gaps } \\
\text { missing }\end{array}$ & $\begin{array}{l}\text { as } \\
\text { data }\end{array}$ & $\begin{array}{l}\text { Gaps a } \\
\text { fifth }\end{array}$ & sthe & & & $\begin{array}{c}\text { Gaps as } \\
\text { missing data }\end{array}$ & $\begin{array}{l}\text { Gaps as the } \\
\text { fifth state }\end{array}$ & Gaps alone & $\begin{array}{c}\text { Gaps as } \\
\text { missing data }\end{array}$ \\
\hline & & 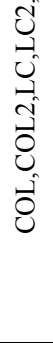 & 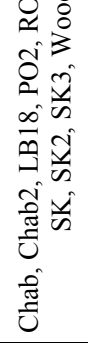 & \begin{tabular}{l}
0 \\
$\mathfrak{\Im}$ \\
\multirow{\Sigma}{\Sigma}{}
\end{tabular} & 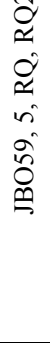 & 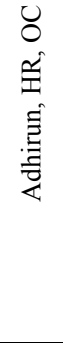 & 这 & $\begin{array}{l}m_{1}^{m} \\
\sum_{i}^{\infty} \\
\stackrel{1}{1}\end{array}$ & 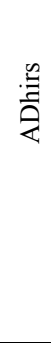 & 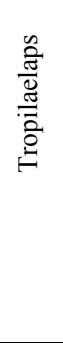 & $\begin{array}{l}\vec{n} \\
0 \\
0 \\
0 \\
2 \\
n \\
0 \\
1 \\
0 \\
0 \\
0 \\
0 \\
0\end{array}$ & ڤ & 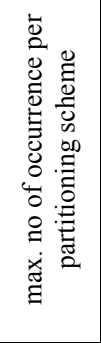 & 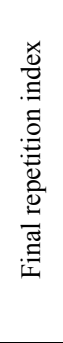 & 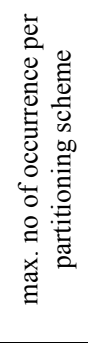 & 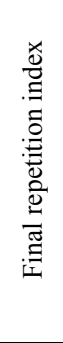 & 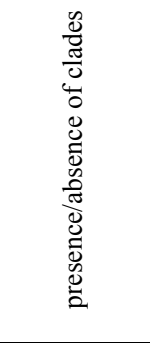 & 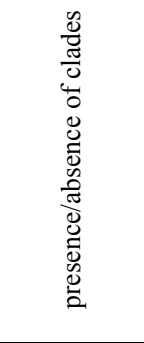 & 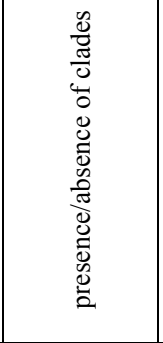 & 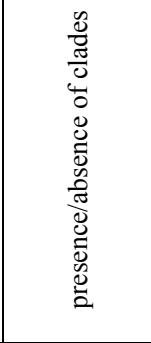 & 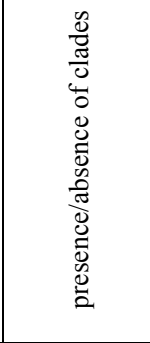 & 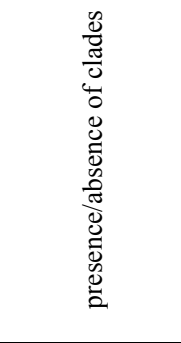 \\
\hline & D. gallinae & $\mathrm{x}$ & $\mathrm{x}$ & & & & & & & & & & 4 & 4 & 4 & 4 & 1 & 1 & 1 & 1 & 1 & 1 \\
\hline & D. gallinae L1 & $\mathrm{x}$ & & & & & & & & & & & 5 & 5 & 5 & 5 & 1 & 1 & 1 & 1 & 1 & 1 \\
\hline & D. gallinae non L1 & & $\mathrm{x}$ & & & & & & & & & & 4 & 4 & 3 & 3 & 1 & 1 & 0 & 0 & 0 & 0 \\
\hline & D. apodis & & & $\mathrm{x}$ & & & & & & & & & 5 & 5 & 5 & 5 & 1 & 1 & 1 & 1 & 1 & 1 \\
\hline & D. hirundinis & & & & & $x$ & & & & & & & 5 & 5 & 5 & 5 & 1 & 1 & 1 & 1 & 1 & 1 \\
\hline & D. carpathicus & & & & $\mathrm{x}$ & & & & & & & & 5 & 5 & 5 & 5 & 1 & 1 & 1 & 1 & 1 & 1 \\
\hline & D. longipes & & & & & & $\mathrm{x}$ & $\mathrm{x}$ & & & & & 4 & 2 & 4 & 3 & 1 & 1 & 0 & 0 & 0 & 0 \\
\hline & O. sylviarum & & & & & & & & & & $\mathrm{x}$ & & 5 & 5 & 5 & 5 & - & - & - & - & - & - \\
\hline & $\mathrm{x}$ & $\mathrm{x}$ & $\mathrm{x}$ & $\mathrm{x}$ & & & & & & & & & 4 & 3 & 4 & 3 & 0 & 0 & 1 & 1 & 1 & 1 \\
\hline & $\mathrm{b}$ & & & & & $\mathrm{x}$ & $\mathrm{x}$ & $\mathrm{x}$ & & & & & 4 & 1 & 4 & 1 & 0 & 0 & 0 & 0 & 0 & 0 \\
\hline & $\mathrm{g}$ & & & & & $\mathrm{x}$ & $\mathrm{x}$ & & & & & & 2 & -2 & 2 & -2 & 0 & 0 & 0 & 0 & 0 & 0 \\
\hline & $\mathrm{d}$ & & & & $\mathrm{x}$ & $\mathrm{x}$ & & & & & & & 3 & -1 & 3 & -1 & 0 & 0 & 0 & 0 & 0 & 0 \\
\hline & $\mathrm{h}$ & & & & $\mathrm{x}$ & $x$ & $x$ & $\mathrm{x}$ & & & & & 3 & 1 & 3 & 1 & 0 & 0 & 0 & 0 & 0 & 0 \\
\hline & $\mathrm{q}$ & & & & $\mathrm{x}$ & $\mathrm{x}$ & $\mathrm{x}$ & $\mathrm{x}$ & $\mathrm{x}$ & & & & 1 & -2 & 0 & - & 1 & 1 & 0 & 0 & 0 & 0 \\
\hline & $\mathbf{a}$ & $\mathrm{x}$ & $\mathrm{x}$ & $\mathrm{x}$ & & & & & $\mathrm{x}$ & & & & 0 & - & 4 & 2 & 0 & 0 & 1 & 1 & 1 & 1 \\
\hline & $\mathrm{m}$ & $\mathrm{x}$ & $\mathrm{x}$ & $\mathrm{x}$ & & $\mathrm{x}$ & $x$ & $\mathrm{x}$ & & & & & 1 & -1 & 2 & -2 & 0 & 0 & 0 & 0 & 0 & 0 \\
\hline & $\mathrm{i}$ & $\mathrm{x}$ & $\mathrm{x}$ & $x$ & $\mathrm{x}$ & $\mathrm{x}$ & $x$ & $\mathrm{x}$ & & & & & 3 & 1 & 2 & -2 & 0 & 0 & 0 & 0 & 0 & 0 \\
\hline & $\mathrm{n}$ & $\mathrm{x}$ & $\mathrm{x}$ & $\mathrm{x}$ & & $x$ & $x$ & $\mathrm{x}$ & $\mathrm{x}$ & & & & 2 & -1 & 2 & -1 & 0 & 0 & 0 & 0 & 0 & 0 \\
\hline & $\mathrm{y}$ & $\mathrm{x}$ & $\mathrm{x}$ & & $\mathrm{x}$ & $\mathrm{x}$ & $\mathrm{x}$ & $\mathrm{x}$ & $\mathrm{x}$ & & & & 1 & -3 & 1 & -3 & 0 & 0 & 0 & 0 & 0 & 0 \\
\hline & $\mathrm{z}$ & & & $\mathrm{x}$ & $\mathrm{x}$ & $\mathrm{x}$ & $\mathrm{x}$ & $\mathrm{x}$ & $\mathrm{x}$ & & & & 0 & - & 0 & - & 1 & 1 & 0 & 0 & 0 & 0 \\
\hline & o & $\mathrm{x}$ & $\mathrm{x}$ & $x$ & $\mathrm{x}$ & $x$ & $\mathrm{x}$ & & $\mathrm{x}$ & & & & 0 & - & 1 & -1 & 0 & 0 & 0 & 1 & 1 & 0 \\
\hline & $\mathrm{p}$ & $\mathrm{x}$ & $\mathrm{x}$ & $\mathrm{x}$ & $\mathrm{x}$ & $\mathrm{x}$ & $\mathrm{x}$ & $x$ & $\mathrm{x}$ & & & & 6 & 6 & 6 & 6 & 0 & 0 & 0 & 0 & 0 & 0 \\
\hline & $\mathrm{r}$ & $\mathrm{x}$ & $\mathrm{x}$ & $\mathrm{x}$ & $\mathrm{x}$ & $\mathrm{x}$ & $\mathrm{x}$ & $\mathrm{x}$ & $\mathrm{x}$ & $x$ & & & 2 & 2 & 0 & - & 0 & 0 & 0 & 0 & 0 & 0 \\
\hline & $\mathrm{s}$ & & & & & & & & & & $\mathrm{x}$ & $\mathrm{x}$ & 6 & 6 & 5 & 5 & 1 & 1 & 1 & 1 & 1 & 1 \\
\hline & $\mathrm{f}$ & $\mathrm{x}$ & $\mathrm{x}$ & $\mathrm{x}$ & $\mathrm{x}$ & & & & $\mathrm{x}$ & & & & 0 & - & 0 & - & 0 & 0 & 0 & 1 & 0 & 1 \\
\hline & $\mathrm{m}$ & & & $\mathrm{x}$ & $\mathrm{x}$ & $\mathrm{x}$ & $\mathrm{x}$ & $\mathrm{x}$ & $\mathrm{x}$ & & & & 0 & - & 0 & - & 1 & 1 & 0 & 0 & 0 & 0 \\
\hline
\end{tabular}




\subsubsection{Specific Characterization Power of Sequences}

Among the six elementary datasets, variability and characterization powers strongly differ, depending on the nature of the considered gene and the genomic location (mitochondrial/nuclear genome), mostly following common rules. Observed divergence percentages are presented in Table 3. These elementary datasets may be split into three groups according to their characterization power:

$1 \mathrm{COI}, 16 \mathrm{~S}$ and Tropomyosin intron $\mathrm{n}$ are very informative at the specific and intraspecific levels, as usually noted in other arthropods.

2 ITS1 and 2 are moderately informative at the specific level and weakly variable within species, contrary to observations of close relatives (Dermanyssoidea: Rhinonyssidae [27,28]), although more similar to other mesostigmatids (Ascoidea: Phytodeiidae [29]).

3 5.8S and Tropomyosin exon $\mathrm{n}$ and $\mathrm{n}+1$ are insufficiently informative at the specific level and do not show any intraspecific variation, as expected.

Table 3. Percentages of divergence in the six elementary datasets defined in Step 1.

\begin{tabular}{|c|c|c|c|c|c|}
\hline & $\begin{array}{l}\text { Between } \\
\text { species }\end{array}$ & $\begin{array}{l}\text { Within } \\
\text { species }\end{array}$ & $\begin{array}{c}\text { Between } \\
\text { D. gallinae } \mathrm{L1} \text { and } \\
\text { D. gallinae non L1 }\end{array}$ & $\begin{array}{l}\text { Between } \\
\text { D. longipes EN and } \\
\text { D. longipes PAS }\end{array}$ & Remarks \\
\hline COI & $9-18 \%$ & $\begin{array}{l}0-5 \% \\
\text { (rarely up } \\
\text { to } 9 \% \text { ) }\end{array}$ & $10-12 \%$ & $5 \%$ & \\
\hline $16 \mathrm{~S}$ & $10-16 \%$ & $0-4 \%$ & $6-7 \%$ & $3 \%$ & \\
\hline $5.8 \mathrm{~S}$ & $0-3 \%$ & $0 \%$ & $0 \%$ & $0 \%$ & $\begin{array}{l}\text { only D. carpathicus and } D \text {. hirsutus differenciated } \\
\text { from each other and from others. }\end{array}$ \\
\hline ITS1 and 2 & $\begin{array}{c}2-5 \% \\
\text { (rarely up to } \\
9 \%)\end{array}$ & $1 \%$ & $3 \%$ & $2 \%$ & $\begin{array}{l}9 \% \text { between } D \text {. hirsutus and other Dermanyssus } \\
\text { species only - More than a half: } 2-3 \%-1 \% \text { in case } \\
\text { between } D \text {. apodis and D. gallinae non L1, and } \\
\text { between } D \text {. hirundinis and } D \text {. longipes EN }\end{array}$ \\
\hline $\begin{array}{l}\text { Tropomyosin } \\
\text { intron } \mathbf{n}\end{array}$ & $8-20 \%$ & $0-6 \%$ & $2-6 \%$ & $4 \%$ & \\
\hline $\begin{array}{c}\text { Tropomyosin } \\
\text { exon } n \text { and } n+1\end{array}$ & cf. remarks & $0 \%$ & $0 \%$ & $0 \%$ & $\begin{array}{l}\text { Very small portion }(25 \mathrm{pb}) .2 \text { point mutations in } D \text {. } \\
\text { apodis vs. other Dermanyssus species. } \\
1-2 \text { point mutations }+1 \text { indel Ornithonyssus vs. } \\
\text { Dermanyssus }\end{array}$ \\
\hline
\end{tabular}

\subsection{Step 2: Multi-Isolate Analyses}

Both COI and Tropomyosin portions have been obtained from 211 individuals. Additionally, COI was also obtained in 41 individuals and Tropomyosin in 16 other individuals. This resulted in 56 different COI haplotypes isolated from five species of Dermanyssus (three in D. longipes, three in D. hirundinis, six in D. carpathicus, four in D. apodis, 35 in D. gallinae, one in D. hirsutus) and 62 different Tropomyosin alleles (five in D. longipes, two in D. hirundinis, seven in D. carpathicus, two 
in D. apodis, 36 in D. gallinae, one in D. hirsutus). See Appendix 1 for detailed informations about individuals and isolates.

\subsubsection{Additive Information about Phylogenetic Interrelationships}

Based on COI and Tropomyosin individual matrices and using Maximum parsimony criterion, by treating successively gaps as missing data and as the fifth state for the second one, three different topologies were obtained (50\% majority rule consensus trees), one of which is recurrently recovered in multi-gene analyses (Top7, Tropomyosin with gaps as missing data) (Appendix 5) and two of which do not exactly match with any of the 38 topologies retained in Step 1 (COI and Tropomyosin with gaps as the fifth state). Indels-only MP analysis reveals a rather strong phylogenetic signal of intronic indels of Tropomyosin, since topologies obtained using indels-only and gaps as missing data generate similar topologies, although slightly less resolved in the former (Appendix 6). Bayesian analyses (Figure 4a) resulted in the same topologies as Maximum parsimony analyses with gaps as missing data, with the exception that one node more is dichotomic with COI (node grouping together non gallinae species) and one node is lost with Tropomyosin (MP node grouping together basal haplotypes Tro_41(Tro_42, 43, 44) (see Appendix 6). With gaps as the fifth state, the MP analysis of Tropomyosin matrix results in the same topology as gaps as missing data Bayesian's with the exception that it contains four more internal dichotomic nodes (Figure 4b). These additional resolved relationships reinforce the scale-like shape of the toplogy. No Bayesian analysis with gaps as the fifth state may be performed using MrBayes.

The monophyly of dermanyssid species under test as well as the monophyly of specific entities D. carpathicus, D. apodis and D. hirundinis are strongly supported. The monophyly of $D$. gallinae is also supported, except in the BA analysis based on COI. D. gallinae special lineage L1 is recovered in all analyses, with support, except in Tropomyosin indels-only MP analysis. The monophyly of D. longipes is recovered only in COI analyses, not in any of the Tropomyosin-based analyses. In the latter, the two isolates of $D$. longipes under test appear as paraphyletic basal entities (see clade o Table 2, Figure 4).

Pairwise relationships between species of Dermanyssus are roughly similar between mitochondrial and nuclear topologies, but an important paraphyly is noted between both loci. Based on both loci, a scale-like topology is retained, in which $D$. apodis appears to be the closest species to D. gallinae, with $D$. hirsutus, then $D$. carpathicus, then $D$. hirundinis and D. longipes as more distant species. But the common ancestor rooting appears to act between $D$. gallinae and D. apodis in mitochondrial analyses, and between $D$. longipes EN and D. longipes PAS in nuclear topologies. 
Figure 4. Haplotypic topologies obtained with COI and Tropomyosin sequences and intraspecific variation. COI (left) and Tropomyosin (right, gaps as missing data) topologies. MrBayes. Bayesian Posterior Probabilities listed at nodes. Mapping of isolates on the topologies is displayed by the names of the simple and focused isolates in italic in front of each haplotype they contain (number of haplotype occurrence in brackets). The comparison of intraspecific variations is above all allowed by mapping the focused isolates sampled in the wild avifauna Dhir F, BER7, GO, IL (names in bold). Within D. gallinae, a $\mathrm{w}$ indicates samples from wild avifauna (other species only from the wild avifauna). Note: here Bayesian topologies are displayed aiming at showing branch length, but one must keep in mind that they resulted in interrelationships similar to MP analyses. Observed host ranges: Passeriformes only: D. longipes PAS (2 Simple Isolates) on sparrows (Passeridae: Passer spp.), D. longipes EN (1 SI) on tits (Paridae: Parus spp.), D. hirundinis French lineage (6 SI) on swallows (Hirundinidae), D. carpathicus (5 SI) on redstarts (Muscicapidae: Phoenicurus sp.) and tits, D. apodis (1 Focused Isolate, $2 \mathrm{SI}$ ) on swifts (Apodidae: Apus sp.); Columbiformes: D. gallinae L1 (1 FI, 6 SI) on pigeons (except 2 dead individuals respectively from a swift and an owl nests); Various bird orders: $D$. gallinae non L1 on Coraciiformes, Passeriformes, Galliformes, Apodiformes of which L3 (1 FI) on starlings (Sturnidae: Sturnus vulgaris).

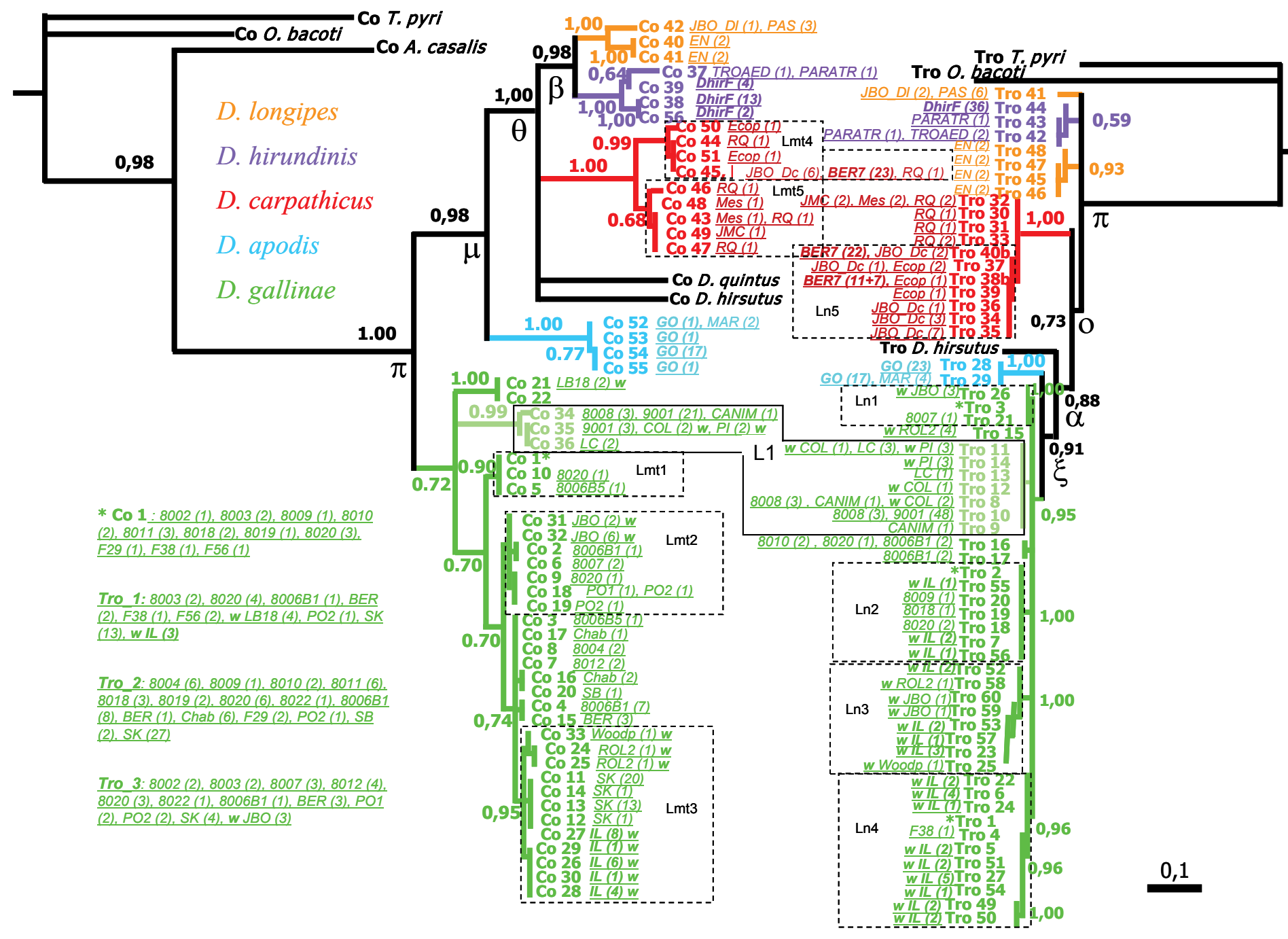




\subsubsection{Differentiation within Previously Delimited Specific Entities}

Intraspecific variability appears very different according to the considered species within Dermanyssus. The simple observed number of haplotypes, along with divergence percentages noted within focused isolates show a marked opposition between D. gallinae and others (Figure 4a). Indeed, when considering focused isolates sampled from nests of wild birds (populations living in comparable environments, i.e., here non anthropized), D. gallinae appears to possess many more Tropomyosin haplotypes, with more divergence than other species under test, (Figure 4a, in bold: focused isolates from wild bird nests in D. apodis, D. carpathicus, D. gallinae and D. hirundinis).

The average number of differences $\mathrm{K}$ in COI haplotypes for focused isolates (Table 4 ) is the lowest in all focused isolates $(0-1.1)$, except in $D$. hirundinis pseudo-isolate DhirF $(\mathrm{K}=4.6)$. This exception was expected since this latter group of individuals was sampled from across France, as opposed to the five true focused isolates. This difference highlights a slight differentiation between isolates of various geographical origins at least in French $D$. hirundinis. The differentiation between isolates within D. gallinae is much sharper (up to $9 \%$ divergence in COI haplotypes between simple and/or focused isolates of $D$. gallinae non L1 from various geographic origins).

The average number of differences $\mathrm{K}$ in Tropomyosin haplotypes for focused isolates is completely null in D. hirundinis pseudo-isolate DhirF and very low in D. apodis and D. carpathicus focused isolates (GO 4.51, BER7 1.83 with gaps considered). In contrast, it is important in D. gallinae focused isolates under test (SK 14.5, IL 32.5, corresponding to up to 7\% divergence), except in D. gallinae L1 (nul as in D. hirundinis).

Both Dermanyssus apodis focused isolate GO and D. carpathicus focused isolate BER7 show low diversity in both mitochondrial and nuclear genes. The inter-isolate intraspecific variation within D. apodis is not clearly estimable based on the present dataset, as only one focused isolate and one simple isolate were sequenced (France). Anyway, the simple isolate involved here of D. apodis (MAR, Center France) provided the same haplotypes as the focused isolate GO in both loci, and three individuals from Corsica provided a COI diverging by only 3-4 nucleotides from GO and MAR (i.e., 0.5-0.7\%; acc. $\mathrm{n}^{\mathrm{o}} \mathrm{FN} 398146$, not included in present analyses). These are sizeable insights of the low variability in COI sequences within $D$. apodis, which appears to be independent of geographical location, whereas a slightly higher divergence is noted in Tropomyosin intron $\mathrm{n}$. As for D. carpathicus, five simple isolates were integrated in analyses in addition to the focused isolate BER7, which reveal relatively low inter-isolate variation in Tropomyosin intron $\mathrm{n}$ and a slightly larger range of values in COI (see branch lengths in Figure 4a). D. gallinae not only possesses by far the highest number of haplotypes in both genes under test in Step 2, but also it shows the highest intraisolate and inter-isolate sequence diversity.

D. gallinae IL and SK show high heterozygosity in Tropomyosin sequences, including numerous allelic indels (Table 4). Heterozygosity is much lower in D. apodis GO, but involves also allelic indels, whereas it is low in D. carpathicus BER7 and does not include any indels (see Table 4). Overall, intraspecific as well as intra-isolate variability in the Tropomyosin locus is very important in D. gallinae non L1, in contrast to D. apodis, D. carpathicus, D. hirundinis and D. gallinae L1 (see Tables 3 and 4). 
Table 4. Information on sequences variability in focused isolates. Genotypic and heterozygosity variability in focused isolates for Tropomyosin exon $n$, intron $n$ and exon $\mathrm{n}+1$ (DnaSP, Arlequin). $\mathrm{n}$ refers to the number of sequences under test, $\mathrm{G}$ to the genotype number, HET(obs) to the observed \% of heterozygozity, Allind to the presence (P)/absence (A) of indels between alleles, $\mathrm{D} \%$ ind to the observed maximum $\%$ of divergence between haplotypes when considering indels, $\mathrm{D} \%$ no ind to the observed maximum $\%$ of divergence between haplotypes whithout considering any indels, $\mathrm{S}$ to the number of segregating sites, Sind to the number of polymorphic/indel/missing sites, $h$ to the allele/haplotype number, $\mathrm{Hd}$ to the haplotype diversity, $\mathrm{K}$ to the average number of differences.

\begin{tabular}{|c|c|c|c|c|c|c|c|c|c|c|c|c|c|c|c|c|c|c|}
\hline \multirow[b]{2}{*}{ 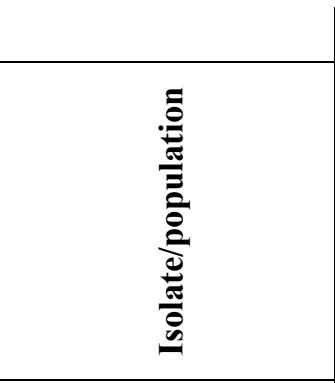 } & \multicolumn{13}{|c|}{ Tropomyosin } & \multicolumn{5}{|c|}{ COI } \\
\hline & $=$ & ט & 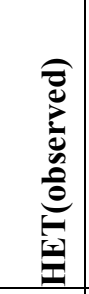 & $\stackrel{\Xi}{\stackrel{\Xi}{E}}$ & 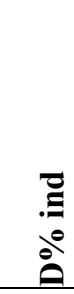 & $\begin{array}{l}. \Xi \\
\stackrel{\Xi}{\Xi} \\
\stackrel{0}{0}\end{array}$ & 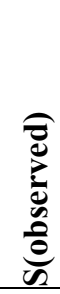 & 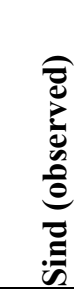 & $=$ & 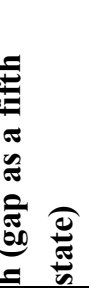 & $\bar{\Xi}$ & 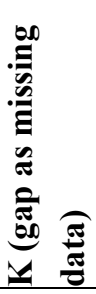 & 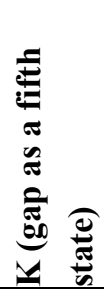 & $=$ & $\infty$ & $=$ & 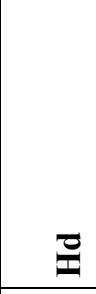 & 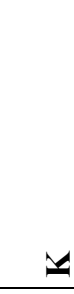 \\
\hline D. gallinae SK & 44 & 4 & 0.41 & $\mathrm{P}$ & 0.03 & 0.02 & 14 & 41 & 3 & 3 & 0.54 & 4.60 & 14.46 & 24 & 3 & 4 & 0.31 & 0.33 \\
\hline D. gallinae IL & 38 & 18 & 0.88 & $\mathrm{P}$ & 0.06 & 0.03 & 37 & 116 & 18 & 18 & 0.94 & 9.56 & 31.88 & 20 & 4 & 5 & 0.74 & 1.06 \\
\hline D. gallinae L1 9001 & 48 & 1 & - & - & 0.00 & 0.00 & 0 & 0 & 1 & 1 & 0.00 & 0.00 & 0.00 & 24 & 8 & 2 & 0.23 & 1.83 \\
\hline D. apodis GO & 40 & 3 & 0.14 & $\mathrm{P}$ & 0.01 & 0.01 & 4 & 9 & 2 & 2 & 0.50 & 2.01 & 4.51 & 20 & 3 & 4 & 0.28 & 0.39 \\
\hline D. carpathicus BER7 & 40 & 5 & 0.70 & $\mathrm{~A}$ & 0.01 & 0.01 & 4 & 4 & 3 & 4 & 0.61 & 1.83 & 1.83 & 24 & 0 & 1 & 0.00 & 0 \\
\hline D. hirundinis DhirF & 36 & 1 & - & - & 0.00 & 0.00 & 0 & 0 & 1 & 1 & 0.00 & 0.00 & 0.00 & 21 & 15 & 4 & 0.53 & 4.59 \\
\hline D. gallinae SK & 44 & 4 & 0.41 & $\mathrm{P}$ & 0.03 & 0.02 & 14 & 41 & 3 & 3 & 0.54 & 4.60 & 14.46 & 24 & 3 & 4 & 0.31 & 0.33 \\
\hline D. gallinae IL & 38 & 18 & 0.88 & $\mathrm{P}$ & 0.06 & 0.03 & 37 & 116 & 18 & 18 & 0.94 & 9.56 & 31.88 & 20 & 4 & 5 & 0.74 & 1.06 \\
\hline D. gallinae L1 9001 & 48 & 1 & - & - & 0.00 & 0.00 & 0 & 0 & 1 & 1 & 0.00 & 0.00 & 0.00 & 24 & 8 & 2 & 0.23 & 1.83 \\
\hline D. apodis GO & 40 & 3 & 0.14 & $\mathrm{P}$ & 0.01 & 0.01 & 4 & 9 & 2 & 2 & 0.50 & 2.01 & 4.51 & 20 & 3 & 4 & 0.28 & 0.39 \\
\hline D. carpathicus BER7 & 40 & 5 & 0.70 & $\mathrm{~A}$ & 0.01 & 0.01 & 4 & 4 & 3 & 4 & 0.61 & 1.83 & 1.83 & 24 & 0 & 1 & 0.00 & 0 \\
\hline D. hirundinis DhirF & 36 & 1 & - & - & 0.00 & 0.00 & 0 & 0 & 1 & 1 & 0.00 & 0.00 & 0.00 & 21 & 15 & 4 & 0.53 & 4.59 \\
\hline
\end{tabular}

The ratio of external branch length / internal branch length in mitochondrial monophyletic groups and the comparison with that of corresponding monophylies shows Tropomyosin is already deeply structured. In the COI gene tree, branch lengths between clades are much longer than intraspecific branch lengths in the species which are strictly encountered in the wild avifauna, but not in the "gallinae complex" [10]. All entities with a ratio $>2$ recovered monophyly in the nuclear topology (e.g., species D. apodis 121.0, D. carpathicus 5.8, D. hirundinis 2.2). On the other hand, none of entities with a ratio $<1.5$ recovered monophyly in the nuclear topology (e.g., D. longipes 1.4), except D. gallinae (1.1). 
Table 5. Mapping isolates in the most supported intraspecific clades. (a) Information on clades. (b) Percentage of common isolates in $\mathrm{mt}$ clade/n clade.

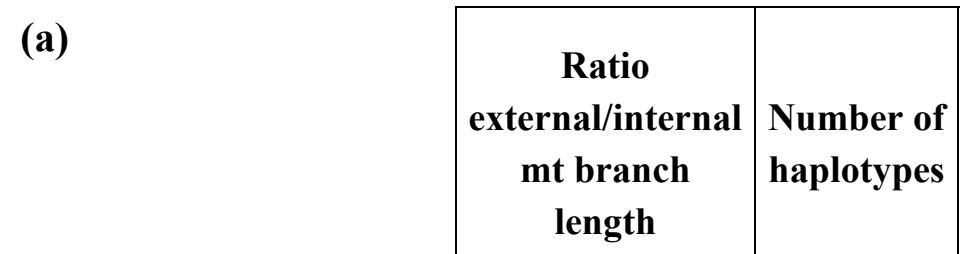

\begin{tabular}{|c|c|c|c|c|}
\hline $\begin{array}{l}\text { Number } \\
\text { of } \\
\text { isolates }\end{array}$ & $\begin{array}{c}\text { Number of } \\
\text { occurrences } \\
\text { (COI } \\
\text { haploid, } \\
\text { Tpm diploid) }\end{array}$ & $\begin{array}{c}\text { Bootstrap } \\
\text { (MP gap } \\
\text { 5th state) }\end{array}$ & $\begin{array}{c}\text { Relative } \\
\text { Bremer } \\
\text { index } \\
\text { (MP gap } \\
\text { 5th state) } \\
\end{array}$ & BPP \\
\hline 6 & 34 & 100 & 100 & 0.99 \\
\hline 12 & 20 & 99 & 100 & 0.9 \\
\hline 6 & 15 & 61 & 100 & 0.69 \\
\hline 4 & 58 & 23 & 50 & 0.95 \\
\hline 4 & 33 & 100 & 100 & 0.99 \\
\hline 3 & 6 & 99 & 100 & 0.68 \\
\hline 6 & 70 & 96 & 92 & 0.97 \\
\hline 13 & 38 & 100 & 88 & 1 \\
\hline 16 & 81 & 97 & 85 & 1 \\
\hline 4 & 12 & 92 & 94 & 1 \\
\hline 11 & 55 & 81 & 83 & 0.96 \\
\hline 3 & 58 & 68 & 100 & 0.79 \\
\hline 6 & 34 & 100 & 100 & 0.99 \\
\hline 12 & 20 & 99 & 100 & 0.9 \\
\hline 6 & 15 & 61 & 100 & 0.69 \\
\hline 4 & 58 & 23 & 50 & 0.95 \\
\hline 4 & 33 & 100 & 100 & 0.99 \\
\hline 3 & 6 & 99 & 100 & 0.68 \\
\hline 6 & 70 & 96 & 92 & 0.97 \\
\hline 13 & 38 & 100 & 88 & 1 \\
\hline 16 & 81 & 97 & 85 & 1 \\
\hline 4 & 12 & 92 & 94 & 1 \\
\hline 11 & 55 & 81 & 83 & 0.96 \\
\hline 3 & 58 & 68 & 100 & 0.79 \\
\hline
\end{tabular}

(b)

\begin{tabular}{|l|llllll|}
\cline { 2 - 7 } \multicolumn{1}{c|}{} & L1 & Ln1 & Ln2 & Ln3 & Ln4 & Ln5 \\
\hline L1 & $100 / 100$ & $0 / 0$ & $0 / 0$ & $0 / 0$ & $0 / 0$ & $0 / 0$ \\
\hline Lmt1 & $0 / 0$ & $25 / 23$ & $58 / 44$ & $0 / 0$ & $33 / 36$ & $0 / 0$ \\
\hline Lmt2 & $0 / 0$ & $100 / 46$ & $50 / 19$ & $17 / 17$ & $50 / 27$ & $0 / 0$ \\
\hline Lmt3 & $0 / 0$ & $50 / 15$ & $50 / 13$ & $75 / 75$ & $50 / 18$ & $0 / 0$ \\
\hline Lmt4 & $0 / 0$ & $0 / 0$ & $0 / 0$ & $0 / 0$ & $0 / 0$ & $75 / 100$ \\
\hline Lmt5 & $0 / 0$ & $0 / 0$ & $0 / 0$ & $0 / 0$ & $0 / 0$ & $0 / 0$ \\
\hline
\end{tabular}


Support values, along with some branch length ratios, draw the attention to some important lineages within $D$. gallinae and $D$. carpathicus. As a result, the examination of the most supported clades led to focus on 3 COI within D. gallinae clades, 2 COI within D. carpathicus clades, $4 \mathrm{Tpm}$ within D. gallinae clades and $1 \mathrm{Tpm}$ within D. carpathicus clade (Table 5a).

By mapping isolates on each supported clade and comparing them between mitochondrial and nuclear topologies (Figure 4a, Table 5b), the special lineage L1 in D. gallinae appears to be completely isolated, with monophyly rather recently reached (short basal branch length in Tpm Bayesian topology). Isolates of special lineage L1 [11] group together in both mitochondrial and nuclear analyses (both MP and BA). However, they do not group as sister to the remaining D. gallinae populations, but arise from within the $D$. gallinae complex. They diverge by $10-13 \%$ from other D. gallinae lineages in COI (1-2\% between each another within L1) and by only two mutational differences (no particular indel) from some other D. gallinae isolates in Tropomyosin. They are more differentiated in Tropomyosin from D. gallinae focused isolates (9001 vs. D. gallinae s. str. Fst 0.44-0.65) than they are between each other (Fst 0.00-0.32), but not as much as from other species (Fst 0.97 against $D$. apodis).

Within species, no other mitochondrial lineages are monophyletic in the nuclear topologies, which are naturally expectable within species and are likely to result from reticulation, as well as recombination, but may also result partly from incomplete lineage sorting. Nevertheless, the mitochondrial clade Lmt3 and the nuclear clade Ln3 share three different isolates sampled in the wild avifauna, in different places and bird host species (two thirds in each clade). This might represent a lineage incompletely isolated. Within D. carpathicus, the clades Lmt4 and Ln5 also share an important portion of isolates and could represent an incompletely isolated lineage.

\section{Discussion}

\subsection{Specific Characterization Power of Sequences}

The results integrating the newly developed nuclear marker Tropomyosin confirm reproductive isolation, and consequently specific status of D. carpathicus, D. hirundinis, D. apodis and D. gallinae. Dermanyssus carpathicus and D. hirsutus appear to be strongly differentiated from each other and from other Dermanyssus species in all genes except the small portions composed of Tropomyosin exon $\mathrm{n}$ and $\mathrm{n}+1$ (see Table 3). Dermanyssus apodis is strongly differentiated from other Dermanyssus species in both mitochondrial datasets and in the nuclear Tropomyosin datasets (both intron and exons), whereas it is very weakly differentiated based on ITS1 and 2 and not differentiated in 5.8S. On the other hand, special lineage L1, although less differentiated than D. apodis in other genes, appears more different in the ITS sequences.

Moreover, although the reproductive isolation of D. longipes isolates seems to be confirmed, this entity is revealed to be diphyletic. Two contradictory clades against $D$. longipes PAS $+\mathrm{EN}$ among the different partitioning schemes tested in Step 1 and a paraphyletic position of D. longipes' Tpm sequences in two well supported clades in the MP analysis of Step 2 with gaps as the fifth state shows that this entity is composed of two different species. The characterization of both these lineages remains imprecise, since they are each represented here by one or two isolates from a single site. However, as noted in Roy et al. [11], sequences of ITS1 and 2 published by Brännström et al. [30] 
from mites sampled in Sweden on different bird species are exactly identical to ITS in D. longipes EN, whereas ITS sequences of $D$. longipes PAS diverge by $2 \%$. Given the very low amount of interspecific divergence in ITS sequences within Dermanyssus, it is very likely that the Swedish isolates belong to the same specific entity as present EN isolates. Of the two D. longipes lineages, D. longipes PAS appears as closer to $D$. hirundinis (Figure $4 \mathrm{~b}$ ). Since this isolate has been sampled near the type locality and in the type host genus, it is to be considered the name bearing species. As a result, the lineage EN will be referred to as Dermanyssus sp. EN in the remaining text.

As for D. gallinae special lineage L1 [11], it appears as an isolated lineage with strong support. The monophyly of this lineage and the gallinae complex is also supported. Nevertheless, its position within the gallinae complex or as a sister to it remains unclear.

Multiple functional copies of EF-1 $\alpha$ have been found in various Arthropoda [31-33] including in some cases both functional and non functional copies in single species [34]. Moreover, Hedin and Maddison [25] have shown the presence of intronless non functional copies among multiple copies in a portion overlapping the region studied here within the genus Habronattus (Aranea: Salticidae) (positions 529-1005 of the H. melpomene CDS). Intronless copies seem to be evolving under relaxed functional constraints. Goetze [34] published a similar report on Crustaceans with exons of putative functional / non functional sequences almost undistinguishable from each other.

In the present datasets, it is worth noting that there is no intron in any of the EF-1 $\alpha$ portions under test, although several other Arthropoda show introns precisely within this portion, especially some Arachnida: between positions 771-772 (836-837 of the whole published sequence), a $167 \mathrm{bp}$ intron is present in Mecaphesa sp. (Arachnida:Araneae:Araneomorphae: Thomisidae, FJ590835), a 124 bp intron in Habronattus (Arachnida: Araneae: Salticidae, AF477231) and a 147 bp intron in Haplochthonius simplex (Arachnida: Acari: Oribatida: Haplochthoniidae, GQ398254). And this position also possesses an intron in some insects (Coleoptera, Hymenoptera) [35] and some crustaceans (Calanoidea: Eucalanidae) [34]. Not to mention that at least two other positions in the present region possess introns in the same crustaceans and in some Diptera [34].

The intronless nature of presently tested sequences may suggest that they are not functional and evolve as pseudogenes. Of course, there are no stop codons in any of obtained sequences, but there are a few non silent mutations either in sequences of clade EF_A or in clade EF_B. And yet EF-1 $\alpha$ has already revealed some difficulties in the application to deeper systematic relationships in Mesostigmata [36], and present inferences show largely unsupported internal relationships within Parasitiformes, including a few incongruences if compared with their phylogenetic relationships robustly established by Klompen et al. [37] based on rRNAs. Here, EF-1 $\alpha$ copies position in the phylogenetic reconstructions of mites based on this gene (Figure 1) demonstrates that gene duplication has occurred anteriorly to the split between Macronyssidae and Dermanyssidae. The date of the duplication may be posterior to the split between dermanyssoid and other Mesostigmata according to the position of double copies in the large phylogenetic reconstruction represented in Figure 1. But further exploration would be needed in order to check this.

All the more, it is not unlikely that other difficulties are generated by multiple copies, including once more non functional ones at a lower taxonomic level within clade EF_B (Figure 1). Indeed, the structure remains unclear, as some sequences of outgroups and of ingroups group together in a distal position. A potentially more recent duplication event may have occurred, but on which present dataset 
is not sufficient to get any explanation, once more likely because of some failure in amplifying all copies. In any case, this gene region does not seem to be appropriate for interspecific investigations within Dermanyssus, nor for interfamily exploration within Dermanyssoidea. And this is in accordance with results of Goetze [34] in Calanoidea (Crustacea), where paralogous gene copies are likely to be problematic for phylogenetic studies, especially at the species level.

\subsection{Phylogenetic Interrelationships at the Specific Level}

In the present study, the biclade topology described by Roy et al. [10] based on mitochondrial DNA, opposing the group of specialist species (D. carpathicus + D. longipes + Dermanyssus $\mathrm{sp}$. EN + D. hirundinis $+D$. apodis + hirsutus-group) to the synanthropic gallinae complex is not supported by most of results (Figures 3 and 4). It appears it is a topology retained solely based on COI alone, only with the enlarged set of isolates in Step 2 (Top5, see Appendix 5), and with rather low support values at internal nodes.

Topologies retained in present multi-gene analyses show a biclade structure with gaps as the fifth state and a semi-biclade structure with gaps as missing data (clades $\xi$ and $\eta$, see Figure 3), but in any cases, the two clades do not oppose non synanthropic species to the synanthropic one: the generalist and synanthropic $D$. gallinae in both greedy consensuses groups with $D$. apodis (clade $\xi$ ), which is a swift specialist and is absent from farms, with correct support (max. occurrences per partitioning scheme 4.0, final repetition index 3).

The rooting of the common ancestor is problematic. When using the enlarged set of isolates (Step 2, multi-isolates analyses), pairwise specific relationships appear similar to multi-gene topologies, but a noticeable paraphyly between mitochondrial and nuclear analyses arises (see Table 2, Figures 3 and 4). In multi-gene greedy consensuses, the position of D. hirsutus remains unclear (basal with gaps as missing data, a sister to the clade $\xi$, i.e., D. gallinae $+D$. apodis) with gaps as the fifth state. At the specific level, in multi-isolate analyses, scale-like topologies are retained, which are mutually and symmetrically paraphyletic, with $D$. gallinae as the basal entity in mt-COI analyses and D. longipes, D. sp. EN and D. hirundinis as the basal entity in nuclear Tpm analyses (Figure 4). In Roy et al. [11], a slight incongruence was vaguely suggested between mitochondrial and nuclear topologies, but the nuclear gene region used in this study was not variable enough to establish it firmly (ITS1-5.8S-ITS2).

The species tree is often not identical to the gene tree [38], due to several potential causes. Reduced effective population size $(\mathrm{Ne})$ in mitochondrial DNA compared to nuclear DNA (a quarter in most sexually reproductive organisms, a third in the present case, due to haplodiploidy) often causes a high mutation rate in mitochondrial DNA resulting in less resolved internal relationship in mitochondrial topologies than in nuclear topologies. As a result, in mitochondrial gene trees, the most recurrent bias is due to homoplasy, and inconsistencies in nuclear gene trees are due to the stochastic effects of lineage sorting. Additionally, interspecific hybridization in some cases may induce reticulation [39].

The choice of a more appropriate gene tree for relationships between species requires some attention. Some authors considered more appropriate mitochondrial markers for inferring phylogenies at the specific level $[40,41]$ but they were dealing with organisms with smaller numbers of generations per year (birds, rodents; D. gallinae in natura, around $15 \mathrm{gen} / \mathrm{y}$, in farms, $>200 \mathrm{gen} / \mathrm{y}$ ), and thus with 
likely reduced mutation rates in both mitochondrial and nuclear genomes. McCracken et al. [39] recommended a balanced approach, taking into account both advantages and flaws due to different effective population size $\mathrm{Ne}$ and considering first whether independent gene trees are adequately resolved and then whether those trees are congruent with the species history.

In the present case, a bias may come from the outgroups: no very close outgroup has been integrated into the analyses, because no individual of Liponyssoides, the only other genus within Dermanyssidae, has been found despite an intense screening of potential hosts. As a result, only other Dermanyssoid families are represented as outgroups (Macronyssidae, Laelapidae, Varroidae). Bayesian topologies reveal important distances between in- and outgroups, which might explain some difficulties in trying to clearly locate the ancestral rooting point. The different genera integrated here as outgroups are rather diverse, and provide good coverage across Dermanyssoidea. Since there are currently no published phylogenetic hypotheses for Dermanyssoidea, it is unknown what is the most appropriate outgroup, which is why it was important to have a diverse and a priori paraphyletic set of outgroups. Additionally, the results indicate that some clades are better, or more reliable than others, and consequently may help for the choice of the most reliable topology: clade $\eta$ (recovered in both greedy summaries in multi-gene analyses, see Table 2, Figure 3) is not recovered in any multi-isolate analyses, and yet its maximum number of occurrence in partitioning schemes is 3.0 and its repetition index is 1 in multi-gene analyses (both gaps as missing data and as the fifth state). On the other hand, clade $\xi$ (D. gallinae $+D$. apodis) receives higher support values in both the multi-gene MP analyses (4.0/3). And yet this clade is present in Tpm multi-isolate analyses, whereas absent in mt-COI multiisolate analysis. Moreover, nuclear results show much less homoplasy (cf. CI and RI in Table 6) than mitochondrial results. And the most supported internal nodes in multi-gene analyses also occur in Tropomyosin-based multi-isolate analyses. Finally, a comparable topology was already suggested based on ITS sequences by Roy et al. [11], but the low amount of DNA divergence in this sequence did not provide enough resolution. It was only vaguely suggested and the few resolved internal nodes were not supported.

The basal position of $D$. hirsutus in the greedy consensus for multi-gene analysis treating gaps as missing data may be due to the lack of indel information present in Tropomyosin intron n. Kawakita et al. [24] have shown that inclusion of intronic gap characters consistently contribute to phylogenetic reconstruction, at least at lower taxonomic levels. Results of indels alone analysis (Appendix 6) strongly suggest that Tpm intronic indels contains important and consistent phylogenetic information and represents interesting complements to the information present in gap-free corresponding intronic portions. And yet, the basal position of D. hirsutus is less supported in gaps as missing data analyses than its median position in gaps as the fifth state (see Figure 3). This strongly suggests that the split between hirsutus group and gallinae group of Moss [42] is not valid. 
Table 6. Consistency Index (as such and excluding uninformative characters) and Retention Index information for "total evidence" and individual MP analyses in Step 1 and for MP analyses in Step 2.

\begin{tabular}{|c|c|c|c|c|c|c|c|}
\hline & & gapmode & No of trees & $\begin{array}{c}\text { Tree } \\
\text { length }\end{array}$ & CI & \begin{tabular}{|c|} 
CI excluding \\
uninformative \\
characters \\
\end{tabular} & RI \\
\hline \multirow{12}{*}{$\begin{array}{l}\text { multi-gene } \\
\text { analyses }\end{array}$} & $6 \mathrm{comb}$ & missing & 1 & 1911 & 0.6787 & 0.6375 & 0.8529 \\
\hline & $6 \mathrm{comb}$ & $5^{\text {th }}$ state & 1 & 2685 & 0.6685 & 0.6421 & 0.8623 \\
\hline & COI (1comb1) & - & 7 & 738 & 0.5068 & 0.4793 & 0.7776 \\
\hline & $16 \mathrm{~S}(1 \mathrm{comb} 2)$ & missing & 5 & 296 & 0.6926 & 0.6527 & 0.8551 \\
\hline & $16 \mathrm{~S}(1 \mathrm{comb} 2)$ & $5^{\text {th }}$ state & 5 & 352 & 0.7131 & 0.6863 & 0.8758 \\
\hline & $5.8 \mathrm{~S}(1 \mathrm{comb} 3)$ & missing & $>1000$ & 48 & 0.875 & 0.8235 & 0.9155 \\
\hline & ITS1_2(1comb4) & missing & 4 & 283 & 0.8375 & 0.784 & 0.891 \\
\hline & ITS1_2 (1comb4) & $5^{\text {th }}$ state & 8 & 376 & 0.8457 & 0.7986 & 0.8854 \\
\hline & TropoINTR (1 comb5) & missing & 18 & 492 & 0.8882 & 0.8721 & 0.9608 \\
\hline & TropoINTR (1 comb5) & $5^{\text {th }}$ state & 126 & 911 & 0.8804 & 0.8685 & 0.9635 \\
\hline & TropoEX (1 comb6) & - & 1 & 3 & 1 & & 1 \\
\hline & $\mathrm{EF}-1 \alpha$ & - & 140 & 165 & 0.8848 & 0.7738 & 0.8545 \\
\hline \multirow{3}{*}{$\begin{array}{l}\text { multi- } \\
\text { isolate } \\
\text { analyses }\end{array}$} & Tropo & missing & 352 & 726 & 0.8278 & 0.7845 & 0.9432 \\
\hline & Tropo & $5^{\text {th }}$ state & $>1000$ & 1371 & 0.8228 & 0.8016 & 0.9494 \\
\hline & COI & - & 868 & 666 & 0.536 & 0.5118 & 0.8657 \\
\hline
\end{tabular}

\subsection{Generalist: A Derived or Ancestral Condition?}

Non gallinae species are more or less specialized, whereas $D$. gallinae has been encountered in nine different bird orders in France by Roy et al. [10]. Dermanyssus apodis and the French isolates of D. hirundinis are rather strict specialists, as exclusively encountered on the genus Apus for the former and in the family Hirundinidae for the latter in France in present study as well as in Roy et al. [10,11]. Looking like intermediate entities on the host specialization point of view, Dermanyssus sp. EN and D. carpathicus are known from two to three different bird families, but both within Passeriformes: Dermanyssus sp. EN on Paridae (in France, present data), Muscicapidae and Sylviidae (in Sweden [30]), D. carpathicus on Paridae and Muscicapidae (present data). The two incompletely isolated lineages within D. carpathicus (Lmt4 and Lmt5) do not appear to be restricted to any of the two passeriform families (see Appendix 1). As for Dermanyssus sp. EN, it is likely to be a moderate specialist, as is D. carpathicus, i.e., parasitizing various bird passeriform families, which are not as conspicuously distant between each other as are D. gallinae's hosts (bird taxonomic considerations follow Peterson's classification [46]), but additional samples are needed to establish it clearly. Finally, not enough isolates of $D$. longipes $s$. str. are available here to estimate the host range of this species.

In all topologies except the multi-isolate COI analyses, a derived state of generalist vs. specialist condition is noted: D. gallinae, the only generalist species in present study [10] is in a distal position. This may indicate, as already shown in the fish ectoparasite Lamellodiscus (Trematoda: Monogenea) [2], as well as in some free-living insects such as bees [3] and springtails (Collembola) [4], that, contrary to usual expectations, the specialist condition does not appear as a "dead-end". Dermanyssus 
gallinae, the generalist species, is, if not the more derived species, at least one of the distal ones with D. apodis, whereas basal positions are occupied by specialist species. And it is worth noting that special lineage L1 of $D$. gallinae has been almost solely encountered in pigeon nests: only two individuals have been found, both dead, in other birds nests (JGC1 and GO8 in Roy et al. [11]), the one in a bird of prey's nest, the second, in a black swift's nest, which is known to be a frequent competitor of pigeons for nest site. Consequently, both these non-pigeon occurrences are likely fortuitous. The precise position of L1 is not absolutely clear, either basal to non L1 D. gallinae, or branching from within $D$. gallinae. In any case, it appears to be currently reproductively isolated and seems to represent a species recently isolated (no morphological difference with non L1 D. gallinae, low divergence based on nuclear loci Tropomyosin intron $\mathrm{n}$ and ITS1 and 2).

The loose relation of Dermanyssus micropredator mites to their host/prey should have led one to expect a fundamentally wider host range in Dermanyssus. And yet, the case of bees' ancestral specialist condition as evidenced by Danforth et al. [3] is the most comparable: despite nectar and pollen collectors do not live on nor develop on their resource-plant, the placement of a paraphyletic Melittidae s.l. at the base of the phylogeny indicates that host-plant specialization is the primitive state.

\subsection{Reticulate Evolution or Gradual Specialization/Speciation?}

Interestingly, the important difference observed in the haplotype variability between species does not seem to be due to the bird's ecology. Apparently, an important intermingling involving a large number of Tpm haplotypes in D. gallinae is noticeable within a colony of starlings (focused isolate IL, Table 4, Figure 4a). But this does not seem to be solely correlated to the bird's ecology, as we noted the exact contrary in $D$. apodis individuals from a colony of swiftlets (focused isolate GO, two Tropomyosin haplotypes) and in D. hirundinis from 6 separate French colonies of barn swallows (focused pseudo-isolate DhirF, one Tropomyosin haplotype). Thus starlings, swiftlets and swallows reuse nests of other pairs in the same colony from one year to another (O. Caparros, CRBPO, MNHN, pers. comm.). This could suggest that $D$. gallinae would have the opportunity to move from one nest to another by phoresy on the bird host (see above). But the ability of $D$. apodis to get transferred by the birds has also been shown by several adult females directly sampled on (flying) hosts [10, 11]. The conspicuous stability of both genes under test in Step $\mathbf{2}$ in D. apodis, D. carpathicus and D. hirundinis strongly contrasts with their variability in D. gallinae, inter-isolate in COI and inter- as well as intraisolate in the nuclear Tropomyosin. This suggests that these represent two types of species that are intrinsically very different, although sister to each other.

This difference between Dermanyssus species might be a consequence of interspecific hybridizations within the $D$. gallinae complex. The monophyly of $D$. gallinae is not doubted here as it is recovered in all topologies. Nevertheless, this is supported by few synapomorphies and the ratio external/internal $\mathrm{mt}$ branch length is below (1.1) the lower limit of mt clades to which monophyletic nuclear clades correspond $(<1.5)$. This could suggest that the date of this coalescence occurred much later than coalescences for other species. But the distance of the coalescent node for D. gallinae to the common ancestor with the closest species D. apodis (considering that the right arrangement is recovered by Tropomyosin multi-isolate topologies) is also by far the shortest (see Bayesian topology, 
Figure 4a). Either the two loci under test in D. apodis, D. hirsutus and D. carpathicus have evolved faster than in $D$. gallinae, or the apparent low rate of evolution within the latter is rather an artifact due to a radiation followed by interbreeding. The latter alternative would appear much more credible since (1) the number of nuclear segregating sites and or indel sites is significantly the highest within D. gallinae focused isolates as opposed to focused isolates or even pseudo-isolates in others species, which is suggestive of the assemblage of formerly highly divergent haplotypes (Table 4), (2) many Tpm genotypes of heterozygous individuals show high divergence percentages within D. gallinae, (3) the specialist lineage L1 with similar stability in both COI and Tpm sequences to other species' is branching from within the widely generalist $D$. gallinae in multi-isolates analyses and basal species are at least moderate specialists, (4) clades Lmt3 and Ln3 share a major part of their isolates and are strongly supported in mitochondrial and nuclear analyses (indels alone and gaps not considered for the nuclear clade Ln3), whereas showing important interbreeding. These two clades could provide evidence of a vestigial completely isolated lineage L3, whose speciation has been in reversion due to subsequent interbreeding.

All that suggests that $D$. gallinae appears as a complex of species, which would have interbred soon after speciation, and just before pre- or post-zygotic incompatibility has been installed. Such a reversal of speciation has been shown to occur in various organisms in case of habitat defragmentation, which induce relaxed divergent selection and increased gene flow because of loss of ecological barriers [47]. Naturally, this potential scenario is no more than a hypothesis, which needs some complementary investigations before being clearly established in D. gallinae. In such a case, the idea evoked by Futuyma and Moreno [1] that specialization and rate of diversification are positively correlated would not be so clearly contradicted by the differential variability depending on specialization within Dermanyssus. The increased molecular diversity in the generalist species would not seem to be due to continuous and intrinsic evolution, but a consequence of the diversification of specialist lineages and consecutive intermingling. It is also worth noting that the only other species with allelic divergence involving some indels in Tropomyosin is D. apodis, the sister to D. gallinae. This suggests that the clade $\xi$ might be particularly prone to hybridize, more than close groups, as it has been shown in some plant groups [48].

Of course, observed differential divergence levels between lineages within D. gallinae complex might suggest a continuum of genetic divergence from sympatric host races to species as recently evidenced in the pea aphid complex [51]. But phylogenetic inferences obtained in present studies, by integrating several sister species and so providing us with a historical scenario, strongly suggests that the gradient of genetic divergence observed here is evolving from weak variability to high variability in the tree of 6 species of Dermanyssus, not the reverse pattern. It's likely the present markers are too slowly evolving to detect new speciation events possibly in process within the gallinae complex. But from the present data it is to be concluded that the generalist condition of $D$. gallinae has been arising from a specialist ancestor. 


\section{Conclusion}

Although reticulate evolution remains difficult to disentangle using phylogenetic tools, comparisons of multi-gene analyses and further analyses of the differentiation in two optimal markers led us to reach some consistent genealogical information at the specific level as well as some insights on more recent isolations and to evidence different evolutionary processes between species.

Intronic nuclear region in Tropomyosin revealed precious phylogenetic signal, at least within Dermanyssus and constitutes a new potentially interesting marker for phylogenetic explorations of Mesostigmata and other arhropods. ITS 1 and 2 do not contain information at intraspecific levels and offer very little interspecific characterization in this genus contrary to some related mite groups. Mitochondrial gene regions are information rich at distal levels, but poorly adapted to resolve internal relationships within Dermanyssus. Finally, as already shown in other arthropods, EF-1alpha does not seem to be appropriate for any phylogenetic/diagnostic exploration within this genus due to some duplication events.

The special lineage L1 seems to represent a cryptic species. The position of D. hirsutus appears to be within the gallinae group of Moss.

The generalist condition revealed to be derived within Dermanyssus, and might result from the hybridization of different specialist entities. The specialist condition of L1 among the generalist lineage complex could represent the sign of its vestigial nature (a remaining lineage isolated during a basal radiation).

Among perspectives, a hypothesis to be tested in the future is: the generalist condition of the gallinae complex might result from hybridizations between different specialist lineages which were each primarily restricted to different and narrow bird groups. Testing predictions obtained with $\mathrm{mt}$ versus nDNA by the mean of an Approximate Bayesian Computation (ABC) method should allow us to check the assumption of hybridization within Dermanyssus. Moreover, it would be interesting to use faster evolving markers such as microsatellites and population assignment as in Peccoud et al. [51] in order to determine whether new host races are not recently isolating from each other within the gallinae complex.

\section{Acknowledgements}

We want to warmly thank the Guest Editor, Alan Templeton, for the invitation to contribute our study to this special issue on "Cladistic Analysis and Molecular Evolution".

We kindly thank S. Lubac (Institut Technique de 1'Aviculture, Lyon, France), F. Boléat et coll. (Ecopôle du Forez, France), O. Kilpinen (Danish Institute of Agricultural Sciences, Lyngby, Denmark), M.W. Sabelis and I. Lesna (IBED, University of Amsterdam, The Netherlands), O. Caparros (CRBPO, MNHN, Paris, France), Damien and Marion Buronfosse for having provided precious mite samples.

Finally, L.R. would like to offer her most sincere thanks for their technical participation to S. Bonnet and N. Guichard N. (LEGTA Saint-Genis-Laval, France), M. Rigaux (IUT A, Université Lyon1, France), S. Merlin (Lycée Jean-Baptiste de la Salle, Lyon, France), S. El Ouartiti (IUT GBGE, Université Jean Monnet, Saint-Etienne, France), G. Lallemand (Lycée des Mandailles, Châteauneuf de Galaure, France). 
This work was supported by the PEP (Pôle d'Expérimentation et de Progrès) Avicole of the Région Rhône-Alpes (France).

\section{References and Notes}

1. Futuyma, D.; Moreno, G. The Evolution of ecological specialization. Ann. Rev. Ecolog. Syst. 1988, 19, 207-233.

2. Desdevises, Y.; Morand, S.; Legendre, P. Evolution and determinants of host specificity in the genus Lamellodiscus (Monogenea). Biol. J. Linn. Soc. 2002, 77, 431-443.

3. Danforth, B.N.; Sipes, S.; Fang, J.; Brady, S.G. The history of early bee diversification based on five genes plus morphology. Proc. Natl. Acad. Sci. USA 2006, 103, 15118-15123.

4. D'Haese, C.A. Were the first springtails semi-aquatic? A phylogenetic approach by means of $28 \mathrm{~S}$ rDNA and optimization alignment. Proc. Royal Soc.: Biol. Sci. 2002, 269, 1143-1151.

5. Zhong, S.; Miller, S.P.; Dykhuizen, D.E.; Dean, A.M. Transcription, Translation, and the Evolution of Specialists and Generalists. Mol. Biol. Evol. 2009, 26, 2661-2678.

6. Kaci-Chaouch, T.; Verneau, O.; Desdevises, Y. Host specificity is linked to intraspecific variability in the genus Lamellodiscus (Monogenea). Parasitology 2008, 135, 607-616.

7. Kuris, A.M.; Lafferty, K.D. Parasite-host modelling meets reality: Adaptive peaks and their ecological attributes. In Evolutionary Biology of Host-Parasite Relationships: Theory Meets Reality; Poulin R., Skorping A., Eds.; Elsevier: Amsterdam, The Netherlands, 2000; pp. 9-26.

8. Moss, W.W. The mite genus Dermanyssus: A survey, with description of Dermanyssus trochilinis, n. sp., and a revised key to the species (Acari: Mesostigmata: Dermanyssidae). J. Med. Entomol. 1978, 14, 627-640.

9. Radovsky, F.J. The evolution of parasitism and the distribution of some Dermanyssoid Mites (Mesostigmata) on vertebrate hosts. In Mites Ecological and Evolutionary Analyses of LifeHistory Patterns; Houck, M.A., Ed.; Chapman \& Hall: New York, NY, USA, 1994.

10. Roy, L.; Dowling, A.P.; Chauve, C.M.; Lesna, I.; Sabelis, M.W.; Buronfosse, T. Molecular phylogenetic assessment of host range in five Dermanyssus species. Exp. Appl. Acarol. 2009, 48, $115-142$.

11. Roy, L.; Dowling, A.P.; Chauve, C.M.; Buronfosse, T. Delimiting species boundaries within Dermanyssus Duges, 1834 (Acari: Dermanyssidae) using a total evidence approach. Mol. Phylogenet. Evol. 2009, 50, 446-470.

12. De Lillo E. A modified method for Eriophyoid mite extraction (Acari: Eriophyoidea). Internat. J. Acarol. 2001, 27, 67-70.

13. Oliver, J.H., Jr. Notes on reproductive behavior in the Dermanyssidae (Acarina Mesostigmata). $J$. Med. Entomol. 1966, 3, 29-35.

14. Hutcheson, H.J.; Oliver, J.H., Jr. Spermiogenesis and reproductive biology of Dermanyssus gallinae (DeGeer) (Parasitiformes: Dermanyssidae). J. Med. Entomol. 1988, 25, 321-330.

15. Edgar, R. MUSCLE: Multiple sequence alignment with high accuracy and high throughput. Nucleic Acids Res. 2004, 32, 1792-1797.

16. Galtier, N.; Gouy, M.; Gautier, C. SEAVIEW and PHYLO_WIN: Two graphic tools for sequence alignment and molecular phylogeny. Comput. Appl. Biosci. 1996, 12, 543-548. 
17. Li, B.; Lecointre, G. Formalizing reliability in the taxonomic congruence approach. Zool. Scr. 2009, 38, 101-112.

18. Rambaut, A.; Drummond, Tracer v1.4; 2009. Available at: http://tree.bio.ed.ac.uk/software/tracer (Accessed on 15 December 2007).

19. Birky, C.W.; Fuerst, P., Maruyama, T. Organelle gene diversity under migration, mutation and drift: Equilibrium expectations, approach to equilibrium, effects of heteroplasmic cells, and comparison to nuclear genes. Genetics 1989, 121, 613-627.

20. Rozas, J.; Rozas, R. DnaSP, DNA sequence polymorphism: An interactive program for estimating population genetics parameters from DNA sequence data. Comput. Appl. Biosci. 1995, 11, 621-625.

21. Excoffier, L.; Laval, G.; Schneider, S. Arlequin (version 3.0): An integrated software package for population genetics data analysis. Evol. Bioinf. Online 2005, 1, 47-50.

22. Black, W.C.; Piesman, J. Phylogeny of hard- and soft-tick taxa (Acari: Ixodida) based on mitochondrial 16S rDNA sequences. Proc. Natl. Acad. Sci. USA 1994, 91, 10034-10038.

23. Sanchis, A.; Michelena, J.M.; Latorre, A.; Quicke, D.L.; Gardenfors, U.; Belshaw, R. The phylogenetic analysis of variable-length sequence data: Elongation factor-1alpha introns in European populations of the parasitoid wasp genus Pauesia (Hymenoptera: Braconidae: Aphidiinae). Mol. Biol. Evol. 2001, 18, 1117-1131.

24. Kawakita, A.; Sota, T.; Ascher, J.S.; Ito, M.; Tanaka, H.; Kato, M. Evolution and phylogenetic utility of alignment gaps within intron sequences of three nuclear genes in bumble bees (Bombus). Mol. Biol. Evol. 2003, 20, 87-92.

25. Hedin, M.C.; Maddison, W.P. Phylogenetic utility and evidence for multiple copies of elongation factor-1alpha in the spider genus Habronattus (Araneae: Salticidae). Mol. Biol. Evol. 2001, 18, $1512-1521$

26. Nisbet, A.J.; Huntley, J.F.; Mackellar, A.; Sparks, N.; McDevitt, R. A house dust mite allergen homologue from poultry red mite Dermanyssus gallinae (De Geer). Parasite Immunol. 2006, 28, 401-405.

27. De Rojas, M., Ubeda, J.M., Cutillas, C., Mora, M.D., Ariza, C., Guevara, D. Utility of ITS1-5.8sITS2 and 16S mitochondrial DNA sequences for species identification and phylogenetic inference within the Rhinonyssus coniventris species complex (Acari: Rhinonyssidae). Parasitol. Res 2007, 100, 1041-1046.

28. De Rojas, M., Mora, M.D., Ubeda, J.M., Cutillas, C., Navajas, M., Guevara, D.C. Phylogenetic relationships in rhinonyssid mites (Acari: Rhinonyssidae) based on ribosomal DNA sequences: Insights for the discrimination of closely related species. Parasitol. Res. 2002, 88, 675-681.

29. Navajas M., Lagnel J., Fauvel G., De Moraes G. Sequence variation of ribosomal internal transcribed spacers (ITS) in commercially important Phytoseiidae mites. Exp. Appl. Acarol. 1999, 23, 851-859.

30. Brännström, S.; Morrison, D.A.; Mattsson, J.G.; Chirico, J. Genetic differences in internal transcribed spacer 1 between Dermanyssus gallinae from wild birds and domestic chickens. Med. Vet. Entomol. 2008, 22, 152-155. 
31. Hovemann, B.; Richter, S.; Walldorf, U.; Cziepluch, C. Two genes encode related cytoplasmic elongation factor 1-alpha (EF-1alpha) in Drosophila melanogaster with continuous and stage specific expression. Nucleic Acids Res. 1988, 16, 3175-3194.

32. Danforth, B.N.; Ji, S. Elongation factor-1 alpha occurs as two copies in bees: Implications for phylogenetic analysis of EF-1 alpha sequences in insects. Mol. Biol. Evol. 1998, 15, 225-235.

33. Williams, S.T.; Knowlton, N.; Weigt, L.A.; Jara, J.A. Evidence for three major clades within the snapping shrimp genus Alpheus inferred from nuclear and mitochondrial gene sequence data. Mol. Phylogenet. Evol. 2001, 20, 375-389.

34. Goetze, E. Elongation factor 1-alpha in marine copepods (Calanoida: Eucalanidae): Phylogenetic utility and unique intron structure. Mol. Phylogenet. Evol. 2006, 40, 880-886.

35. Brady, S.G.; Danforth, B.N. Recent intron gain in elongation factor-1alpha of colletid bees (Hymenoptera: Colletidae). Mol. Biol. Evol. 2004, 21, 691-696.

36. Klompen, H. Preliminary Assessment of the utility of elongation factor-1alpha in elucidating relationships among basal Mesostigmata. Exp. Appl. Acarol. 2000, 24, 805-820.

37. Klompen, H.; Lekveishvili, M.; Black, W.C. Phylogeny of parasitiform mites (Acari) based on rRNA. Mol. Phylogenet. Evol. 2007, 43, 936-951.

38. Nichols, R. Gene trees and species trees are not the same. Trends Ecol. Evol. 2000, 16, 358-364.

39. McCracken, K.; Sorenson, M. Is homoplasy or lineage sorting the source of incongruent mtDNA and nuclear gene trees in the stiff-tailed ducks (Nomonyx-oxyura)? Syst. Biol. 2005, 54, 35-55.

40. Moore, W.S. Inferring phylogenies from the mt-DNA variation: Mitochondrial-gene trees versus nuclear-gene trees. Evolution 1995, 49, 718-726.

41. Michaux, J.R.; Chevret, P.; Filippucci, M.G.; Macholan, M. Phylogeny of the genus Apodemus with a special emphasis on the subgenus Sylvaemus using the nuclear IRBP gene and two mitochondrial markers: Cytochrome b and 12SrRNA. Mol. Phylogenet. Evol. 2002, 23, 123-136

42. Moss, W.W. An Illustrated Key to the Species of the Acarine Genus Dermanyssus (Mesostigmata: Laelapoidea: Dermanyssidae). J. Med. Entomol. 1968, 1, 67-84.

43. Peterson, A. Zoonomen Nomenclatural Data, Version 8.07; Available at: http://www.zoonomen.net/avtax/frame.html (Accessed on 24 November 2009).

44. Seehausen, O.; Takimoto, G.; Roy, D.; Jokela, J. Speciation reversal and biodiversity dynamics with hybridization in changing environments. Mol. Ecol. 2007, 17, 30-44.

45. Whitney, K.D.; Ahern, J.R.; Campbell L.G. Hybridization-prone plant families do not generate more invasive species. Biol. Invas. 2009, 11, 1205-1215.

46. Peccoud, J.; Ollivier, A.; Plantegenest, M.; Simon, J.C. A continuum of genetic divergence from sympatric host races to species in the pea aphid complex. Proc. Natl. Acad. Sci. USA 2009, 106, 7495-7500.

\section{List of Electronic Supplementary Information (ESI)}

ISOL_TRO1 (nexus DNA alignment of Tropomyosin partial exon n, complete intron n, partial exon $\mathrm{n}+1$ upon which the matrix of encoded indels has been established - see Appendix z3).

Appendix 1 (Sampling and EMBL information for the populations under test in present study)

Appendix 2 (Primer sequences) 
Appendix 3 (matrix of encoded Indels in Tropomyosin)

Appendix 4 (The different partitioning schemes for the multi-gene analyses and estimation of repetition index)

Appendix 5 (The different topologies obtained at the specific level within Dermanyssus in multigene analyses and in multi-isolate analyses)

Appendix 6 (MP topologies obtained in Step 2 not shown in Figure 4)

(C) 2010 by the authors; licensee Molecular Diversity Preservation International, Basel, Switzerland. This article is an open-access article distributed under the terms and conditions of the Creative Commons Attribution license (http://creativecommons.org/licenses/by/3.0/). 